\title{
Testing the symmetry of a dependence structure with a characteristic function
}

https://doi.org/10.1515/demo-2018-0019

Received February 26, 2018; accepted November 16, 2018

\begin{abstract}
This paper proposes competing procedures to the tests of symmetry for bivariate copulas of Genest, Nešlehová and Quessy (2012). To this end, the null hypothesis of symmetry is expressed in terms of the copula characteristic function that uniquely determines the copula of a given bivariate population with continuous marginal distributions. Then, test statistics based on $L_{2}$ weighted distances computed from an empirical version of the copula characteristic function are proposed. Their asymptotic behavior is derived under the null hypothesis as well as under general alternatives. In particular, it is established that these rank statistics behave asymptotically as first-order degenerate V-statistics under the null hypothesis and this large-sample representation is exploited in order to provide suitably adapted multiplier bootstrap versions for the computation of p-values. The simulations that are reported show that the new tests are more powerful than the competing methods based on the empirical copula introduced by Genest, Nešlehová and Quessy (2012).
\end{abstract}

Keywords: Asymmetric copulas, degenerate V-statistics, multiplier bootstrap, rank statistics

\section{Introduction}

A random pair $\left(X_{1}, X_{2}\right)$ is exchangeable if $\left(X_{1}, X_{2}\right)$ and $\left(X_{2}, X_{1}\right)$ have the same joint distribution, which translates into $\mathbb{P}\left(X_{1} \leq x_{1}, X_{2} \leq x_{2}\right)=\mathbb{P}\left(X_{2} \leq x_{1}, X_{1} \leq x_{2}\right)$ for all $\left(x_{1}, x_{2}\right) \in \mathbb{R}^{2}$. Assuming continuous marginals for $X_{1}$ and $X_{2}$ and invoking Sklar's Theorem, the random pair $\left(X_{1}, X_{2}\right)$ is exchangeable if and only if $X_{1}$ and $X_{2}$ are identically distributed and if its unique copula $C$ is symmetric in the sense that $C\left(u_{1}, u_{2}\right)=C\left(u_{2}, u_{1}\right)$ for all $\left(u_{1}, u_{2}\right) \in(0,1)^{2}$.

When the goal is to find an appropriate dependence model for a given random pair, it may be a relevant preliminary step to check if their underlying bivariate copula is symmetric without assuming that the marginal distributions are the same. Formally, the null hypothesis of copula symmetry can be expressed as

$$
\mathbb{H}_{0}: C\left(u_{1}, u_{2}\right)=C\left(u_{2}, u_{1}\right), \quad \forall\left(u_{1}, u_{2}\right) \in(0,1)^{2} .
$$

The latter can be tested against the alternative $\mathbb{H}_{1}$ that there exists $\mathcal{A} \subseteq(0,1)^{2}$ with non-null Lebesgue measure such that $C\left(u_{1}, u_{2}\right) \neq C\left(u_{2}, u_{1}\right)$ for all $\left(u_{1}, u_{2}\right) \in \mathcal{A}$. Consistent tests for choosing between $\mathbb{H}_{0}$ and $\mathbb{H}_{1}$ based on the empirical copula have been proposed by [5].

The aim of this paper is to develop competing procedures to those of [5] by making use of the so-called copula characteristic function. The latter has recently been introduced as a well-performing alternative to inference procedures based on empirical copulas; see [2] for radial symmetry tests, [1] for goodness-of-fit procedures and [16] for general homogeneity hypotheses. Specifically, the copula characteristic function associated to a copula $C$ is defined for $i^{2}=-1$ and $\left(U_{1}, U_{2}\right) \sim C$ by

$$
\Psi_{C}\left(t_{1}, t_{2}\right)=\mathrm{E}\left\{e^{i\left(t_{1} U_{1}+t_{2} U_{2}\right)}\right\}, \quad\left(t_{1}, t_{2}\right) \in \mathbb{R}^{2} .
$$

Tarik Bahraoui: University of Sao Paulo, Brazil, E-mail: Tarikb@ime.usp.br

Taoufik Bouezmarni: Université de Sherbrooke, Canada, E-mail: Taoufik.Bouezmarni@usherbrooke.ca

*Corresponding Author: Jean-François Quessy: Université du Québec à Trois-Rivières, Canada,

E-mail: Jean-Francois.Quessy@uqtr.ca 
Because the copula characteristic function completely characterizes $C$, i.e. $C$ can be recovered from the knowledge of $\Psi_{C}$, the null hypothesis of copula symmetry can be reformulated as

$$
\mathbb{H}_{0}: \Psi_{C}\left(t_{1}, t_{2}\right)=\Psi_{C}\left(t_{2}, t_{1}\right), \quad \forall\left(t_{1}, t_{2}\right) \in \mathbb{R}^{2} .
$$

The alternative hypothesis can be reformulated in the same spirit, i.e. $\mathbb{H}_{1}$ means that there exists a subset $\mathcal{B} \subseteq \mathbb{R}^{2}$ with non-null Lebesgue measure such that $\Psi_{C}\left(t_{1}, t_{2}\right)-\Psi_{C}\left(t_{2}, t_{1}\right) \neq 0$ for $\left(t_{1}, t_{2}\right) \in \mathcal{B}$. New test statistics can then be based on an empirical version of the difference $\Psi_{C}\left(t_{1}, t_{2}\right)-\Psi_{C}\left(t_{2}, t_{1}\right)$. Doing so will provide competing procedures to the distribution-oriented methodologies of [5].

The paper is organized as follows. In Section 2, the new test statistics for bivariate copula symmetry are introduced and their asymptotic distributions under the null and alternative hypotheses are established. The validity of a multiplier bootstrap methodology and the consistency of the tests are proved in Section 3; compact formulas for their implementation are provided as well. The results of a simulation study on the size and power of the newly proposed tests are presented in Section 4; comparisons are also made with the competing tests of [5]. Finally, Section 5 briefly discusses on possible extensions of the presented methodology. The proofs of the main results and supplementary material are postponed to two appendices.

\section{New statistics for bivariate copula symmetry}

\subsection{A general class of test statistics}

Let $\left(X_{11}, X_{12}\right), \ldots,\left(X_{n 1}, X_{n 2}\right)$ be independent copies of a random pair $\left(X_{1}, X_{2}\right)$ whose marginal distributions $F_{1}(x)=\mathbb{P}\left(X_{1} \leq x\right)$ and $F_{2}(x)=\mathbb{P}\left(X_{2} \leq x\right)$ are continuous and whose unique copula is $C$. If $F_{1}$ and $F_{2}$ were completely specified, one could work with independent copies $\left(U_{11}, U_{12}\right), \ldots,\left(U_{n 1}, U_{n 2}\right)$ of $\left(U_{1}, U_{2}\right) \sim C$, where $U_{j 1}=F_{1}\left(X_{j 1}\right)$ and $U_{j 2}=F_{2}\left(X_{j 2}\right)$ for all $j \in\{1, \ldots, n\}$.

However, in most of the applications, $F_{1}$ and $F_{2}$ are unknown, so that the pair $\left(U_{1}, U_{2}\right)$ is unobservable. In order to estimate $\Psi_{C}$, one then has to rely on the pairs of pseudo-observations $\left(\widehat{U}_{11}, \widehat{U}_{12}\right), \ldots,\left(\widehat{U}_{n 1}, \widehat{U}_{n 2}\right)$, where for $\widehat{F}_{1}$ and $\widehat{F}_{2}$ being the (re-scaled) empirical distribution functions, $\widehat{U}_{j 1}=\widehat{F}_{1}\left(X_{j 1}\right)$ and $\widehat{U}_{j 2}=\widehat{F}_{2}\left(X_{j 2}\right)$. Specifically,

$$
\widehat{U}_{j 1}=\frac{1}{n+1} \sum_{k=1}^{n} \mathbb{I}\left(X_{k 1} \leq X_{j 1}\right) \quad \text { and } \quad \widehat{U}_{j 2}=\frac{1}{n+1} \sum_{k=1}^{n} \mathbb{I}\left(X_{k 2} \leq X_{j 2}\right) .
$$

A natural empirical version of $\Psi_{C}$ is then given for $\left(t_{1}, t_{2}\right) \in \mathbb{R}^{2}$ by

$$
\Psi_{n}\left(t_{1}, t_{2}\right)=\frac{1}{n} \sum_{j=1}^{n} e^{i\left(t_{1} \widehat{U}_{j 1}+t_{2} \widehat{U}_{j 2}\right)} .
$$

Since under the null hypothesis of copula symmetry, $\Psi_{C}\left(t_{1}, t_{2}\right)=\Psi_{C}\left(t_{2}, t_{1}\right)$ for all $\left(t_{1}, t_{2}\right) \in \mathbb{R}^{2}$, it seems natural to base tests of $\mathbb{H}_{0}$ against $\mathbb{H}_{1}$ on some functional distance between $\Psi_{C}\left(t_{1}, t_{2}\right)$ and $\Psi_{C}\left(t_{2}, t_{1}\right)$. To this end, take a nonnegative and integrable weight function $\omega: \mathbb{R}^{2} \rightarrow \mathbb{R}$ and define the statistic

$$
S_{n, \omega}=\int_{\mathbb{R}^{2}}\left|\Psi_{n}\left(t_{1}, t_{2}\right)-\Psi_{n}\left(t_{2}, t_{1}\right)\right|^{2} \omega\left(t_{1}, t_{2}\right) \mathrm{d} t_{1} \mathrm{~d} t_{2},
$$

where $|z|=\sqrt{x^{2}+y^{2}}$ is the modulus of the complex number $z=x+i y$. Note that the integrability of $\omega$ ensures that the integral in the definition of $S_{n, \omega}$ exists. Also observe that because the integrand in the definition of $S_{n, \omega}$ is symmetric, one can assume without loss of generality that the weight function is symmetric, i.e. $\omega\left(t_{1}, t_{2}\right)=\omega\left(t_{2}, t_{1}\right)$ for all $\left(t_{1}, t_{2}\right) \in \mathbb{R}^{2}$. Indeed, if an asymmetric weight function $\omega$ is used, then it is easy to show that $S_{n, \omega}=S_{n, \widetilde{\omega}}$, where $\widetilde{\omega}\left(t_{1}, t_{2}\right)=\left\{\omega\left(t_{1}, t_{2}\right)+\omega\left(t_{2}, t_{1}\right)\right\} / 2$ is symmetric.

The following lemma provides an explicit formula for $S_{n, \omega}$. Before stating it, define for $\mathbf{a}=\left(a_{1}, a_{2}\right) \in$ $[0,1]^{2}$ and $\mathbf{b}=\left(b_{1}, b_{2}\right) \in[0,1]^{2}$ the function

$$
B_{\omega}(\mathbf{a}, \mathbf{b})=2 \widetilde{B}_{\omega}\left(a_{1}-b_{1}, a_{2}-b_{2}\right)-2 \widetilde{B}_{\omega}\left(a_{2}-b_{1}, a_{1}-b_{2}\right),
$$


where $\widetilde{B}_{\omega}\left(a_{1}, a_{2}\right)=\int_{\mathbb{R}^{2}} \cos \left(t_{1} a_{1}+t_{2} a_{2}\right) \omega\left(t_{1}, t_{2}\right) \mathrm{d} t_{1} \mathrm{~d} t_{2}$.

Lemma 2.1. For $B_{\omega}$ defined in Equation (1), one has for $\widehat{\mathbf{U}}_{j}=\left(\widehat{U}_{j 1}, \widehat{U}_{j 2}\right)$ that

$$
S_{n, \omega}=\frac{1}{n^{2}} \sum_{j, k=1}^{n} B_{\omega}\left(\widehat{\mathbf{U}}_{j}, \widehat{\mathbf{U}}_{k}\right) \text {. }
$$

\subsection{Large-sample distribution of $S_{n, \omega}$}

The asymptotic distributions of $S_{n, \omega}$ under the null hypothesis $\mathbb{H}_{0}$ of copula symmetry, as well as under the alternative hypothesis $\mathbb{H}_{1}$, are established in this section. Note that the form of $S_{n, \omega}$ in Lemma 2.1 is similar to a V-statistic with kernel $B_{\omega}$ of degree two, but computed from the ranks of the observations. As will be shown, this has a significant impact on its asymptotic behavior if one compares to the case when the marginal distributions are known, i.e. when $\mathbf{U}_{1}, \ldots, \mathbf{U}_{n}$ are observable. In that situation, the test statistic would be $S_{n, \omega}^{\prime}=\left(1 / n^{2}\right) \sum_{j, k=1}^{n} B_{\omega}\left(\mathbf{U}_{j}, \mathbf{U}_{k}\right)$ and its asymptotic behavior would easily be derived from classical results on V-statistics.

It will first be shown that the use of the ranks in the definition of $S_{n, \omega}$ entails that the latter is asymptotically equivalent to a V-statistic of order four. Before stating the result, let $\mathbf{u}=\left(u_{1}, u_{2}\right) \in[0,1]^{2}$ and define for each $\mathbf{t}=\left(t_{1}, t_{2}\right) \in \mathbb{R}^{2}$ the function

$$
\mathcal{L}_{\mathbf{t}}(\mathbf{u})=\exp \left\{i\left(t_{1} u_{1}+t_{2} u_{2}\right)\right\}-\exp \left\{i\left(t_{2} u_{1}+t_{1} u_{2}\right)\right\}
$$

Then, letting $\mathcal{L}_{\mathbf{t}}^{[1]}(\mathbf{u})=\partial \mathcal{L}_{\mathbf{t}}(\mathbf{u}) / \partial u_{1}$ and $\mathcal{L}_{\mathbf{t}}^{[2]}(\mathbf{u})=\partial \mathcal{L}_{\mathbf{t}}(\mathbf{u}) / \partial u_{2}$ be the partial derivatives of $\mathcal{L}_{\mathbf{t}}$, define $\Omega_{\mathbf{t}}\left(\mathbf{u}_{1}, \mathbf{u}_{2}\right)=\left\{\widetilde{\Omega}_{\mathbf{t}}\left(\mathbf{u}_{1}, \mathbf{u}_{2}\right)+\widetilde{\Omega}_{\mathbf{t}}\left(\mathbf{u}_{2}, \mathbf{u}_{1}\right)\right\} / 2$ as the symmetrization of the function

$$
\widetilde{\Omega}_{\mathbf{t}}\left(\mathbf{u}_{1}, \mathbf{u}_{2}\right)=\mathcal{L}_{\mathbf{t}}\left(\mathbf{u}_{1}\right)+\left\{\mathbb{I}\left(u_{21} \leq u_{11}\right)-u_{11}\right\} \mathcal{L}_{\mathbf{t}}^{[1]}\left(\mathbf{u}_{1}\right)+\left\{\mathbb{I}\left(u_{22} \leq u_{12}\right)-u_{12}\right\} \mathcal{L}_{\mathbf{t}}^{[2]}\left(\mathbf{u}_{1}\right) .
$$

Finally, for $\mathbf{u}_{\ell}=\left(u_{\ell 1}, u_{\ell 2}\right), \ell \in\{1,2,3,4\}$, let

$$
\begin{aligned}
\Phi_{\omega}\left(\mathbf{u}_{1}, \mathbf{u}_{2}, \mathbf{u}_{3}, \mathbf{u}_{4}\right)= & \frac{1}{3} \int_{\mathbb{R}^{2}} \Omega_{\mathbf{t}}\left(\mathbf{u}_{1}, \mathbf{u}_{2}\right) \Omega_{-\mathbf{t}}\left(\mathbf{u}_{3}, \mathbf{u}_{4}\right) \omega(\mathbf{t}) \mathrm{d} \mathbf{t}+\frac{1}{3} \int_{\mathbb{R}^{2}} \Omega_{\mathbf{t}}\left(\mathbf{u}_{1}, \mathbf{u}_{3}\right) \Omega_{-\mathbf{t}}\left(\mathbf{u}_{2}, \mathbf{u}_{4}\right) \omega(\mathbf{t}) \mathrm{d} \mathbf{t} \\
& +\frac{1}{3} \int_{\mathbb{R}^{2}} \Omega_{\mathbf{t}}\left(\mathbf{u}_{1}, \mathbf{u}_{4}\right) \Omega_{-\mathbf{t}}\left(\mathbf{u}_{2}, \mathbf{u}_{3}\right) \omega(\mathbf{t}) \mathrm{d} \mathbf{t} .
\end{aligned}
$$

The result may now be stated.

Proposition 2.2. Let $\left(X_{11}, X_{12}\right), \ldots,\left(X_{n 1}, X_{n 2}\right)$ be i.i.d. from a bivariate distribution function with continuous marginal distributions $F_{1}$ and $F_{2}$ and copula $C$. If $\omega: \mathbb{R}^{2} \rightarrow \mathbb{R}$ is a non-negative and integrable function that satisfies $\int_{\mathbb{R}^{2}}\left(t_{1}+t_{2}\right)^{4} \omega\left(t_{1}, t_{2}\right) \mathrm{d} t_{1} \mathrm{~d} t_{2}<\infty$, then

$$
S_{n, \omega}=\frac{1}{n^{4}} \sum_{j, k, j^{\prime}, k^{\prime}=1}^{n} \Phi_{\omega}\left(\mathbf{U}_{j}, \mathbf{U}_{k}, \mathbf{U}_{j^{\prime}}, \mathbf{U}_{k^{\prime}}\right)+o_{\mathbb{P}}(1 / n),
$$

where $\mathbf{U}_{j}=\left(F_{1}\left(X_{j 1}\right), F_{2}\left(X_{j 2}\right)\right)$ for each $j \in\{1, \ldots, n\}$.

The next two results establish the asymptotic distributions of $S_{n, \omega}$ under the null hypothesis and under the alternative. As shown in the proofs of these results (see Appendix A.3 and Appendix A.4), the derivation of these large-sample representations is mainly based on the fact that the kernel $\Phi_{\omega}$ is first order degenerate if and only if the null hypothesis $\mathbb{H}_{0}$ holds (under an additional mild condition on the positivity of $\omega$ ). In other words, for $\mathbf{U}_{2}, \mathbf{U}_{3}, \mathbf{U}_{4}$ i.i.d. $C$, then $\mathrm{E}\left\{\Phi_{\omega}\left(\mathbf{u}_{1}, \mathbf{U}_{2}, \mathbf{U}_{3}, \mathbf{U}_{4}\right)\right\}=0$ if and only if $C$ is a symmetric copula. 
Proposition 2.3. Assume the same conditions as in Proposition 2.2. Under the null hypothesis $\mathbb{H}_{0}$ of a symmetric copula $C$,

$$
S_{n, \omega}=\frac{1}{n^{2}} \sum_{j, k=1}^{n} Y_{C, \omega}\left(\mathbf{U}_{j}, \mathbf{U}_{k}\right)+o_{\mathbb{P}}(1 / n),
$$

where for $\Lambda_{C, \mathbf{t}}(\mathbf{u})=\mathcal{L}_{\mathbf{t}}(\mathbf{u})+\mathrm{E}\left\{\mathbb{I}\left(U_{1} \geq u_{1}\right) \mathcal{L}_{\mathbf{t}}^{[1]}(\mathbf{U})+\mathbb{I}\left(U_{2} \geq u_{2}\right) \mathcal{L}_{\mathbf{t}}^{[2]}(\mathbf{U})\right\}, \mathbf{U}=\left(U_{1}, U_{2}\right) \sim C$,

$$
Y_{C, \omega}\left(\mathbf{u}_{1}, \mathbf{u}_{2}\right)=\int_{\mathbb{R}^{2}} \Lambda_{C, \mathbf{t}}\left(\mathbf{u}_{1}\right) \Lambda_{C,-\mathbf{t}}\left(\mathbf{u}_{2}\right) \omega(\mathbf{t}) \mathrm{d} \mathbf{t} .
$$

Hence, $n S_{n, \omega}$ converges in distribution to a random variable that admits the representation $\mathbb{S}_{C, \omega}=$ $\mathrm{E}\left\{Y_{C, \omega}(\mathbf{U}, \mathbf{U})\right\}+\sum_{\kappa=1}^{\infty} \eta_{\kappa}\left(Z_{\kappa}^{2}-1\right)$, where $\left\{Z_{\kappa}\right\}_{\kappa=1}^{\infty}$ are i.i.d. $\mathcal{N}(0,1)$ and $\left\{\eta_{\kappa}\right\}_{\kappa=1}^{\infty}$ are the eigenvalues of $h(\mathbf{u}) \mapsto$ $\mathrm{E}\left\{Y_{C, \omega}(\mathbf{u}, \mathbf{U}) h(\mathbf{U})\right\}$.

One closes this section by investigating the large-sample behavior of $S_{n, \omega}$ under the alternative. Unlike the case when $C$ is symmetric, the kernel $\Phi_{\omega}$ is non-degenerate under the alternative hypothesis $\mathbb{H}_{1}$. As shown in the next result, this entails the asymptotic normality of $S_{n, \omega}$. Before stating it, let

$$
\mu_{C, \omega}=\int_{\mathbb{R}^{2}}\left|\Psi_{C}\left(t_{1}, t_{2}\right)-\Psi_{C}\left(t_{2}, t_{1}\right)\right|^{2} \omega\left(t_{1}, t_{2}\right) \mathrm{d} t_{1} \mathrm{~d} t_{2} .
$$

Proposition 2.4. Assume the same conditions as in Proposition 2.2, and suppose that $\omega$ is strictly positive, except maybe on a subset of $\mathbb{R}^{2}$ of Lebesgue measure zero. Under the alternative hypothesis $\mathbb{H}_{1}$ of an asymmetric copula $C, \sqrt{n}\left(S_{n, \omega}-\mu_{C, \omega}\right)$ converges weakly to the normal distribution with mean zero and variance $\sigma_{C, \omega}^{2}=$ $16 \operatorname{Var}\left\{\Phi_{\omega}^{(1)}\left(\mathbf{U}_{1}\right)\right\}$, where $\Phi_{\omega}^{(1)}(\mathbf{u})=\mathrm{E}\left\{\Phi_{\omega}\left(\mathbf{u}, \mathbf{U}_{2}, \mathbf{U}_{3}, \mathbf{U}_{4}\right)\right\}$ and $\mathbf{U}_{1}, \mathbf{U}_{2}, \mathbf{U}_{3}, \mathbf{U}_{4}$ are i.i.d. $C$.

\section{Performing the tests}

\subsection{Multiplier bootstrap versions}

Proposition 2.3 and Proposition 2.4 suggest that a consistent test would consist in rejecting $\mathbb{H}_{0}$ in favour of $\mathbb{H}_{1}$ for large values of $n S_{n, \omega}$. However, obtaining a formal decision rule is challenging here since on one part, the limit distribution of $n S_{n, \omega}$ under the null hypothesis has a complicated expression, and in addition, the latter depends on an unspecified symmetric copula $C$. For these reasons, one must rely on a re-sampling strategy for the computation of asymptotically valid p-values. The method that is proposed here is based on the multiplier bootstrap. The latter has been extensively used for the testing of shape hypotheses involving copulas, as is the case here.

In the sequel, a multiplier bootstrap sample consists of independent and positive random variables $\xi_{1}, \ldots, \xi_{n}$ such that for each $j \in\{1, \ldots, n\}, \mathrm{E}\left(\xi_{j}\right)=\operatorname{Var}\left(\xi_{j}\right)=1$. Following [4], as long as the pair $\left(U_{1}, U_{2}\right)$ is observable from $\left(U_{11}, U_{12}\right), \ldots,\left(U_{n 1}, U_{n 2}\right)$ i.i.d. $C$, a multiplier version of $n S_{n, \omega}$ would be given by

$$
\widehat{S}_{n, \omega}^{\prime}=\frac{1}{n} \sum_{j, k=1}^{n}\left(\frac{\xi_{j}}{\bar{\xi}}-1\right)\left(\frac{\xi_{k}}{\bar{\xi}}-1\right) Y_{C, \omega}\left(\mathbf{U}_{j}, \mathbf{U}_{k}\right),
$$

where $\bar{\xi}=\left(\xi_{1}+\cdots+\xi_{n}\right) / n$ and $Y_{C, \omega}$ is defined in (6). However, since the marginal distributions are unknown, the pair $\left(U_{1}, U_{2}\right)$ is unobservable. Moreover, the kernel $Y_{C, \omega}$ depends on the unspecified copula $C$. For these reasons, an alternate version of $\widehat{S}_{n, \omega}^{\prime}$ is proposed where the unobserved pairs are replaced by the pseudoobservations $\widehat{\mathbf{U}}_{1}, \ldots, \widehat{\mathbf{U}}_{n}$ and where $Y_{C, \omega}$ is estimated by

$$
\widehat{Y}_{\omega}\left(\mathbf{u}_{1}, \mathbf{u}_{2}\right)=\int_{\mathbb{R}^{2}} \widehat{\Lambda}_{\mathbf{t}}\left(\mathbf{u}_{1}\right) \widehat{\Lambda}_{-\mathbf{t}}\left(\mathbf{u}_{2}\right) \omega(\mathbf{t}) \mathrm{d} \mathbf{t},
$$


where for $\mathbf{u}=\left(u_{1}, u_{2}\right)$ and $\mathbf{t} \in \mathbb{R}^{2}$, the function $\Lambda_{\mathbf{t}}$ described in Proposition 2.3 is estimated by

$$
\widehat{\Lambda}_{\mathbf{t}}(\mathbf{u})=\mathcal{L}_{\mathbf{t}}(\mathbf{u})+\frac{1}{n} \sum_{k=1}^{n}\left\{\mathbb{I}\left(\widehat{U}_{k 1} \geq u_{1}\right) \mathcal{L}_{\mathbf{t}}^{[1]}\left(\widehat{\mathbf{U}}_{k}\right)+\mathbb{I}\left(\widehat{U}_{k 2} \geq u_{2}\right) \mathcal{L}_{\mathbf{t}}^{[2]}\left(\widehat{\mathbf{U}}_{k}\right)\right\}
$$

The proposed multiplier version of $n S_{n, \omega}$ is then

$$
\widehat{S}_{n, \omega}=\frac{1}{n} \sum_{j, k=1}^{n}\left(\frac{\xi_{j}}{\bar{\xi}}-1\right)\left(\frac{\xi_{k}}{\bar{\xi}}-1\right) \widehat{Y}_{\omega}\left(\widehat{\mathbf{U}}_{j}, \widehat{\mathbf{U}}_{k}\right) .
$$

In practice, one considers $N$ multiplier replicates $\widehat{S}_{n, \omega}^{(1)}, \ldots, \widehat{S}_{n, \omega}^{(N)}$ of $n S_{n, \omega}$ based on $N$ independent multiplier bootstrap samples. The estimated p-value of the test based on $S_{n, \omega}$ is then

$$
\widehat{\mathrm{PV}}_{\omega}=\frac{1}{N} \sum_{\ell=1}^{N} \mathbb{I}\left(\widehat{S}_{n, \omega}^{(\ell)}>n S_{n, \omega}\right),
$$

and $\mathbb{H}_{0}$ is rejected at level $\alpha$ if $\widehat{\mathrm{PV}}_{\omega}<\alpha$. The next proposition establishes the asymptotic validity of this resampling procedure.

Proposition 3.1. Assume the same conditions as in Proposition 2.2 and let $\mathbb{P}^{\star}$ be the probability measure conditional on the sample. Then

$$
\sup _{r \in \mathbb{R}^{+}}\left|\mathbb{P}^{\star}\left(\widehat{S}_{n, \omega} \leq r\right)-\mathbb{P}\left(\widetilde{\mathbb{S}}_{C, \omega} \leq r\right)\right| \stackrel{\mathbb{P}}{\longrightarrow} 0,
$$

where for $Y_{C, \omega}$ as defined in Proposition 2.3, the random variable $\widetilde{\mathbb{S}}_{C, \omega}$ admits the representation $\widetilde{\mathbb{S}}_{C, \omega}=$ $\mathrm{E}\left\{Y_{C, \omega}(\mathbf{U}, \mathbf{U})\right\}+\sum_{\kappa=1}^{\infty} \eta_{\kappa}\left(Z_{\kappa}^{2}-1\right)$, with $\left\{Z_{\kappa}\right\}_{\kappa=1}^{\infty}$ being i.i.d. $\mathcal{N}(0,1)$ and $\left\{\eta_{\kappa}\right\}_{\kappa=1}^{\infty}$ are the eigenvalues of $h(\mathbf{u}) \mapsto$ $\mathrm{E}\left\{Y_{C, \omega}(\mathbf{u}, \mathbf{U}) h(\mathbf{U})\right\}$.

It is worth noting that Proposition 3.1 holds both under $\mathbb{H}_{0}$ and $\mathbb{H}_{1}$. On one side, in view of the asymptotic representation of $n S_{n, \omega}$ in Proposition 2.3 and the representation of the random variable $\widetilde{\mathbb{S}}_{C, \omega}$ in Proposition 3.1, one deduces that $\widehat{S}_{n, \omega}^{(1)}, \ldots, \widehat{S}_{n, \omega}^{(N)}$ are asymptotically independent copies of $n S_{n, \omega}$ under $\mathbb{H}_{0}$. Hence, the test maintains its prescribed level asymptotically. On the other side, in view of Proposition $2.4, S_{n, \omega} \rightarrow \mu_{C, \omega}>0$ in probability under $\mathbb{H}_{1}$ as long as $\omega$ is strictly positive (except maybe on a subset of $\mathbb{R}^{2}$ of Lebesgue measure zero). Because $\widetilde{\mathbb{S}}_{C, \omega}$ has a non-degenerate distribution, this entails that $\widehat{\mathrm{PV}}_{\omega} \approx \mathbb{P}\left(\widetilde{\mathbb{S}}_{C, \omega}>n \mu_{C, \omega}\right) \stackrel{n \rightarrow \infty}{\rightarrow} 0$. In other words, the test based on $S_{n, \omega}$ is consistent against any alternative to $\mathbb{H}_{0}$.

\subsection{Formulas for the implementation of the tests}

The multiplier versions $\widehat{S}_{n, \omega}^{(1)}, \ldots, \widehat{S}_{n, \omega}^{(N)}$ of $S_{n, \omega}$ can be conveniently computed from operations on matrices. First of all, let $D_{\omega} \in \mathbb{R}^{n \times n}$ be such that

$$
\left(D_{\omega}\right)_{j k}=\widehat{Y}_{\omega}\left(\widehat{\mathbf{U}}_{j}, \widehat{\mathbf{U}}_{k}\right)=\int_{\mathbb{R}^{2}} \widehat{\Lambda}_{\mathbf{t}}\left(\widehat{\mathbf{U}}_{j}\right) \widehat{\Lambda}_{-\mathbf{t}}\left(\widehat{\mathbf{U}}_{k}\right) \omega(\mathbf{t}) \mathrm{d} \mathbf{t} .
$$

Let also $\Delta^{(\ell)}=\left(\Delta_{1}^{(\ell)}, \ldots, \Delta_{n}^{(\ell)}\right), \ell \in\{1, \ldots, N\}$, be independent vectors of multiplier variables, where $\Delta_{j}^{(\ell)}=$ $\left(\xi_{j}^{(\ell)} / \bar{\xi}^{(\ell)}\right)-1$. From Equation (7), one obtains

$$
\widehat{S}_{n, \omega}^{(\ell)}=\frac{1}{n} \Delta^{(\ell)} D_{\omega}\left(\Delta^{(\ell)}\right)^{\top} .
$$

The main computational effort concerns the matrix $D_{\omega}$, since once the latter is computed from the data, $\widehat{S}_{n, \omega}^{(1)}, \ldots, \widehat{S}_{n, \omega}^{(N)}$ can be computed very quickly. This allows for the generation of a large number $N$ of multiplier replicates for the estimation of the $\mathrm{p}$-value. The following lemma provides with a useful formula for the computation of $D_{\omega}$. 
Lemma 3.2. Let $I_{1}, I_{2} \in \mathbb{R}^{n \times n}$ be such that $\left(I_{1}\right)_{j k}=\mathbb{I}\left(\widehat{U}_{j 1} \leq \widehat{U}_{k 1}\right)$ and $\left(I_{2}\right)_{j k}=\mathbb{I}\left(\widehat{U}_{j 2} \leq \widehat{U}_{k 2}\right)$. Also, for $B_{\omega}^{[\ell]}\left(\mathbf{u}_{1}, \mathbf{u}_{2}\right)=\partial B_{\omega}\left(\mathbf{u}_{1}, \mathbf{u}_{2}\right) / \partial u_{1 \ell}$ and $B_{\omega}^{\left[\ell \ell^{\prime}\right]}\left(\mathbf{u}_{1}, \mathbf{u}_{2}\right)=\partial^{2} B_{\omega}\left(\mathbf{u}_{1}, \mathbf{u}_{2}\right) / \partial u_{1 \ell} \partial u_{2 \ell^{\prime}}$, define $D_{\omega, 0}, D_{\omega, \ell}, D_{\omega, \ell \ell^{\prime}} \in$ $\mathbb{R}^{n \times n}$ such that $\left(D_{\omega, 0}\right)_{j k}=B_{\omega}\left(\widehat{\mathbf{U}}_{j}, \widehat{\mathbf{U}}_{k}\right),\left(D_{\omega, \ell}\right)_{j k}=B_{\omega}^{[\ell]}\left(\widehat{\mathbf{U}}_{j}, \widehat{\mathbf{U}}_{k}\right)$ and $\left(D_{\omega, \ell \ell^{\prime}}\right)_{j k}=B_{\omega}^{\left[\ell \ell^{\prime}\right]}\left(\widehat{\mathbf{U}}_{j}, \widehat{\mathbf{U}}_{k}\right)$. Then,

$$
\begin{aligned}
D_{\omega}= & D_{\omega, 0}+\frac{1}{n}\left(I_{1} D_{\omega, 1}+I_{2} D_{\omega, 2}+\left(I_{1} D_{\omega, 1}\right)^{\top}+\left(I_{2} D_{\omega, 2}\right)^{\top}\right) \\
& +\frac{1}{n^{2}}\left(I_{1} D_{\omega, 11} I_{1}^{\top}+I_{1} D_{\omega, 12} I_{2}^{\top}+I_{2} D_{\omega, 21} I_{1}^{\top}+I_{2} D_{\omega, 22} I_{2}^{\top}\right) .
\end{aligned}
$$

In the statistical literature on characteristic function tests, it is of common use to consider a weight function that is based on a product of densities, i.e. $\omega\left(t_{1}, t_{2}\right)=g\left(t_{1}\right) g\left(t_{2}\right)$, where $g$ is a density on $\mathbb{R}$; see [8], for instance. In that case, one obtains using the trigonometric identity $\cos (x+y)=\cos x \cos y-\sin x \sin y$ that

$$
\begin{aligned}
\widetilde{B}_{\omega}\left(a_{1}, a_{2}\right) & =\int_{\mathbb{R}^{2}} \cos \left(t_{1} a_{1}+t_{2} a_{2}\right) g\left(t_{1}\right) g\left(t_{2}\right) \mathrm{d} t_{1} \mathrm{~d} t_{2} \\
& =\int_{\mathbb{R}^{2}}\left\{\cos \left(t_{1} a_{1}\right) \cos \left(t_{2} a_{2}\right)-\sin \left(t_{1} a_{1}\right) \sin \left(t_{2} a_{2}\right)\right\} g\left(t_{1}\right) g\left(t_{2}\right) \mathrm{d} t_{1} \mathrm{~d} t_{2} \\
& =\int_{\mathbb{R}} \cos \left(t_{1} a_{1}\right) g\left(t_{1}\right) \mathrm{d} t_{1} \int_{\mathbb{R}} \cos \left(t_{2} a_{2}\right) g\left(t_{2}\right) \mathrm{d} t_{2}-\int_{\mathbb{R}} \sin \left(t_{1} a_{1}\right) g\left(t_{1}\right) \mathrm{d} t_{1} \int_{\mathbb{R}} \sin \left(t_{2} a_{2}\right) g\left(t_{2}\right) \mathrm{d} t_{2} \\
& =\beta_{g}^{\mathrm{Re}}\left(a_{1}\right) \beta_{g}^{\mathrm{Re}}\left(a_{2}\right)-\beta_{g}^{\mathrm{Im}}\left(a_{1}\right) \beta_{g}^{\mathrm{Im}}\left(a_{2}\right),
\end{aligned}
$$

where $\beta_{g}^{\mathrm{Re}}$ and $\beta_{g}^{\mathrm{Im}}$ are the real and imaginary parts, respectively, of the characteristic function $\beta_{g}$ of $g$. If one further assumes that $g$ is symmetric around zero, then $\widetilde{B}_{\omega}\left(a_{1}, a_{2}\right)=\beta_{g}\left(a_{1}\right) \beta_{g}\left(a_{2}\right)$ and Equation (1) entails

$$
B_{\omega}(\mathbf{a}, \mathbf{b}) \propto \beta_{g}\left(a_{1}-b_{1}\right) \beta_{g}\left(a_{2}-b_{2}\right)-\beta_{g}\left(a_{2}-b_{1}\right) \beta_{g}\left(a_{1}-b_{2}\right) .
$$

Letting $\mathbf{a}^{\top}=\left(a_{2}, a_{1}\right)$, it follows easily that

$$
B_{\omega}^{[1]}(\mathbf{a}, \mathbf{b})=\beta_{g}^{\prime}\left(a_{1}-b_{1}\right) \beta_{g}\left(a_{2}-b_{2}\right)-\beta_{g}^{\prime}\left(a_{1}-b_{2}\right) \beta_{g}\left(a_{2}-b_{1}\right)
$$

and $B_{\omega}^{[2]}(\mathbf{a}, \mathbf{b})=B_{\omega}^{[1]}\left(\mathbf{a}^{\top}, \mathbf{b}^{\top}\right)$. Moreover,

$$
B_{\omega}^{[11]}(\mathbf{a}, \mathbf{b})=-\beta_{g}^{\prime \prime}\left(a_{1}-b_{1}\right) \beta_{g}\left(a_{2}-b_{2}\right)+\beta_{g}^{\prime}\left(a_{1}-b_{2}\right) \beta_{g}^{\prime}\left(a_{2}-b_{1}\right)
$$

and

$$
B_{\omega}^{[12]}(\mathbf{a}, \mathbf{b})=-\beta_{g}^{\prime}\left(a_{1}-b_{1}\right) \beta_{g}^{\prime}\left(a_{2}-b_{2}\right)+\beta_{g}^{\prime \prime}\left(a_{1}-b_{2}\right) \beta_{g}\left(a_{2}-b_{1}\right) .
$$

Finally, $B_{\omega}^{[21]}(\mathbf{a}, \mathbf{b})=B_{\omega}^{[12]}\left(\mathbf{a}^{\top}, \mathbf{b}^{\top}\right)$ and $B_{\omega}^{[22]}(\mathbf{a}, \mathbf{b})=B_{\omega}^{[11]}\left(\mathbf{a}^{\top}, \mathbf{b}^{\top}\right)$.

\section{Investigation of the performance of the tests}

\subsection{General considerations}

This section investigates the sampling properties of the tests based on $S_{n, \omega}$ when the weight function $\omega$ is a product of standard normal and double-exponential densities, i.e. for $\sigma>0$ being a smoothing parameter,

$$
\omega_{\sigma}^{\mathrm{N}}\left(t_{1}, t_{2}\right)=e^{-\left(t_{1}^{2}+t_{2}^{2}\right) /\left(2 \sigma^{2}\right)} \text { and } \omega_{\sigma}^{\mathrm{DE}}\left(t_{1}, t_{2}\right)=e^{-\left(\left|t_{1}\right|+\left|t_{2}\right|\right) / \sigma} .
$$

These weight functions are integrable and satisfy $\int_{\mathbb{R}^{2}}\left(t_{1}+t_{2}\right)^{4} \omega\left(t_{1}, t_{2}\right) \mathrm{d} t_{1} \mathrm{~d} t_{2}<\infty$, as required in Propositions 2.3, 2.4 and 3.1. They are also strictly positive on their respective domain, ensuring the consistency of the related test statistics. Moreover, since these densities are symmetric, $B_{\omega}$ can be computed from the formula in (8). In the case of the normal density, i.e. $g=\phi$, one has $\beta_{g}(a)=\phi(a), \beta_{g}^{\prime}(a)=-a \phi(a)$ and $\beta^{\prime \prime}(a)=\left(a^{2}-1\right) \phi(a)$, while in the case of the double-exponential density,

$$
\beta_{g}(a)=\frac{1}{4+a^{2}}, \quad \beta_{g}^{\prime}(a)=-\frac{2 a}{\left(4+a^{2}\right)^{2}} \quad \text { and } \quad \beta_{g}^{\prime \prime}(a)=\frac{6 a^{2}-8}{\left(4+a^{2}\right)^{3}} .
$$


The test statistics corresponding to $\omega_{\sigma}^{\mathrm{N}}$ and $\omega_{\sigma}^{\mathrm{DE}}$ will be referred respectively to $S_{n, \sigma}^{\mathrm{N}}$ and $S_{n, \sigma}^{\mathrm{DE}}$ in the sequel. Of course, many other choices for $\omega$ are possible. However, some experiences not shown here indicate that using products of density is generally better than considering for instance bivariate densities. Moreover, as will be seen from the simulations that are reported, it is the value of the smoothing parameter $\sigma$ that mostly influences the performance of the tests, rather than a particular choice of $g$.

For the computation of $\mathrm{p}$-values, the multiplier random variables $\xi_{1}, \ldots, \xi_{n}$ are drawn from the Exponential distribution with mean one; this version of the multiplier method is sometimes called the Bayesian bootstrap; see [10], for instance. Of course, other choices of distributions for the multipliers are possible, but based on the authors' experience, it has a negligible influence on the results. The number of bootstrap replicates has been set to $N=1000$ and the estimated probabilities of rejection are based on 1000 Monte-Carlo replicates. All tests have been performed at the $5 \%$ level.

\subsection{Size of the tests}

An important step before the study of the power of the tests is to assess their ability to keep their nominal level under $\mathbb{H}_{0}$. In our context, this is somewhat equivalent in checking the performance of the multiplier bootstrap method outside the asymptotic framework. To this end, the models that will be considered under $\mathbb{H}_{0}$ are the Clayton, Gumbel, Normal and centered chi-square copulas. Their expressions are given respectively by

$$
\begin{aligned}
C_{\theta}^{C \ell}\left(u_{1}, u_{2}\right) & =\left(u_{1}^{-\theta}+u_{2}^{-\theta}-1\right)^{-1 / \theta}, \quad \theta>0, \\
C_{\theta}^{\mathrm{Gu}}\left(u_{1}, u_{2}\right) & =\exp \left\{-\left(\left|\ln u_{1}\right|^{1 /(1-\theta)}+\left|\ln u_{2}\right|^{1 /(1-\theta)}\right)^{1-\theta}\right\}, \quad \theta \in[0,1], \\
C_{\theta}^{\mathrm{N}}\left(u_{1}, u_{2}\right) & =\Phi_{\theta}\left\{\Phi^{-1}\left(u_{1}\right), \Phi^{-1}\left(u_{2}\right)\right\}, \quad \theta \in[-1,1], \\
C_{\theta}^{\chi}\left(u_{1}, u_{2}\right) & =2\left\{C_{\theta}^{\mathrm{N}}\left(\frac{1+u_{1}}{2}, \frac{1+u_{2}}{2}\right)-C_{\theta}^{\mathrm{N}}\left(\frac{1+u_{1}}{2}, \frac{1-u_{2}}{2}\right)\right\}-u_{2},
\end{aligned}
$$

where $\Phi_{\theta}$ is the cumulative distribution function (cdf) of the standard bivariate Normal distribution with correlation $\theta$ and $\Phi^{-1}$ is the inverse of the standard univariate normal cdf. The reader is referred to the books by [14] and [13] for more details on the first three of these models. For the construction and properties of the general family of chi-square copulas, see [18].

The results on the estimated probabilities of rejection are found in Table 1 for $n \in\{100,250,500\}$ and $\sigma \in\{1,3,5\}$. In order to ease the interpretation of the results, the models have been parameterized in terms of their associated Kendall's tau, defined for a given copula $C$ by

$$
\tau_{C}=4 \int_{0}^{1} \int_{0}^{1} C\left(u_{1}, u_{2}\right) \mathrm{d} C\left(u_{1}, u_{2}\right)-1 .
$$

Looking at Table 1, one can say that when $n=100$ and $n=250$, the tests are generally good at keeping their nominal level, except when $\tau_{C}=.75$, where the tests are conservative; this comment apply to the four copula models that have been considered and whatever the value of $\sigma$. This behavior is typical of the multiplier method in a copula context under high levels of dependence; see [3]. For $n=500$, however, all tests are close to their nominal level, as expected from the asymptotic theory.

\subsection{Choice of the bandwidth parameter}

As observed by [2] in the case of tests of multivariate radial symmetry, the value of the parameter $\sigma$ may have a significant influence on the power of tests based on the copula characteristic function. As it may well be the case here, the power of the tests based on $S_{n, \sigma}^{\mathrm{N}}$ and $S_{n, \sigma}^{\mathrm{DE}}$ as a function of $\sigma$ will first be investigated in 
Table 1: Percentages of rejection, as estimated from 1000 replicates, for the tests of bivariate copula symmetry based on the characteristic function statistics $S_{n, \sigma}^{\mathrm{N}}$ and $S_{n, \sigma}^{\mathrm{DE}}$ when $\sigma \in\{1,3,5\}$ under the symmetric Clayton, Gumbel, Normal and chisquare copulas; upper panel: $n=100$; middle panel: $n=250$; bottom panel: $n=500$

\begin{tabular}{|c|c|c|c|c|c|c|c|}
\hline Copula & $\tau_{C}$ & $S_{n, 1}^{\mathrm{N}}$ & $S_{n, 3}^{\mathrm{N}}$ & $S_{n, 5}^{\mathrm{N}}$ & $S_{n, 1}^{\mathrm{DE}}$ & $S_{n, 3}^{\mathrm{DE}}$ & $S_{n, 5}^{\mathrm{DE}}$ \\
\hline & .25 & 3.9 & 4.3 & 2.5 & 4.0 & 3.9 & 3.5 \\
\hline \multirow[t]{3}{*}{ Clayton } & .50 & 3.0 & 3.5 & 2.9 & 2.2 & 3.6 & 1.9 \\
\hline & .75 & 0.1 & 0.5 & 0.5 & 0.0 & 0.7 & 0.3 \\
\hline & .25 & 4.3 & 4.1 & 3.6 & 4.2 & 3.9 & 4.0 \\
\hline \multirow[t]{3}{*}{ Gumbel } & .50 & 1.9 & 3.3 & 2.8 & 2.1 & 2.3 & 2.2 \\
\hline & .75 & 0.4 & 0.9 & 0.5 & 0.0 & 0.3 & 0.6 \\
\hline & .25 & 4.9 & 4.7 & 3.5 & 3.1 & 4.1 & 4.0 \\
\hline \multirow[t]{3}{*}{ Normal } & .50 & 2.6 & 3.2 & 2.2 & 2.1 & 3.0 & 2.9 \\
\hline & .75 & 0.1 & 0.6 & 0.5 & 0.0 & 0.2 & 0.3 \\
\hline & .25 & 5.9 & 5.1 & 2.6 & 4.0 & 4.2 & 4.3 \\
\hline \multirow[t]{3}{*}{ Chi-square } & .50 & 2.8 & 2.9 & 1.8 & 1.5 & 3.0 & 2.1 \\
\hline & .75 & 0.1 & 0.6 & 0.4 & 0.0 & 0.0 & 0.1 \\
\hline & .25 & 5.7 & 5.3 & 4.6 & 5.7 & 5.6 & 4.2 \\
\hline \multirow[t]{3}{*}{ Clayton } & .50 & 3.3 & 3.8 & 3.5 & 3.6 & 4.1 & 3.9 \\
\hline & .75 & 2.1 & 2.7 & 2.8 & 1.9 & 3.3 & 2.5 \\
\hline & .25 & 4.6 & 5.2 & 4.6 & 3.6 & 4.4 & 4.6 \\
\hline \multirow[t]{3}{*}{ Gumbel } & .50 & 3.4 & 5.5 & 3.4 & 4.0 & 4.5 & 4.0 \\
\hline & .75 & 2.2 & 1.8 & 3.0 & 2.0 & 2.1 & 1.6 \\
\hline & .25 & 5.5 & 4.7 & 4.0 & 4.0 & 4.1 & 4.2 \\
\hline \multirow[t]{3}{*}{ Normal } & .50 & 6.0 & 4.4 & 3.4 & 5.6 & 3.2 & 3.8 \\
\hline & .75 & 3.1 & 2.7 & 2.3 & 1.6 & 0.8 & 1.0 \\
\hline & .25 & 4.8 & 5.2 & 4.1 & 5.0 & 4.9 & 3.6 \\
\hline \multirow[t]{3}{*}{ Chi-square } & .50 & 4.0 & 3.6 & 3.5 & 2.9 & 3.2 & 2.8 \\
\hline & .75 & 1.9 & 2.4 & 2.4 & 1.5 & 1.9 & 0.7 \\
\hline & .25 & 5.2 & 5.7 & 4.5 & 4.7 & 4.7 & 4.6 \\
\hline \multirow[t]{3}{*}{ Clayton } & .50 & 4.5 & 4.9 & 2.6 & 5.2 & 5.6 & 3.6 \\
\hline & .75 & 4.4 & 5.3 & 4.2 & 2.2 & 3.4 & 2.9 \\
\hline & .25 & 4.9 & 5.5 & 3.5 & 4.0 & 5.2 & 5.0 \\
\hline \multirow[t]{3}{*}{ Gumbel } & .50 & 4.1 & 5.3 & 4.8 & 4.1 & 4.3 & 4.1 \\
\hline & .75 & 2.9 & 4.2 & 3.1 & 3.5 & 2.9 & 4.5 \\
\hline & .25 & 4.6 & 5.5 & 6.0 & 4.5 & 5.0 & 3.4 \\
\hline \multirow[t]{3}{*}{ Normal } & .50 & 4.4 & 4.5 & 4.3 & 5.0 & 5.2 & 3.7 \\
\hline & .75 & 3.2 & 4.0 & 4.0 & 2.7 & 2.7 & 2.8 \\
\hline & .25 & 5.7 & 4.9 & 5.3 & 5.0 & 5.1 & 4.4 \\
\hline \multirow[t]{2}{*}{ Chi-square } & .50 & 4.5 & 4.0 & 4.8 & 3.5 & 4.2 & 4.4 \\
\hline & .75 & 3.4 & 3.2 & 2.9 & 4.1 & 4.5 & 3.0 \\
\hline
\end{tabular}


order to identify some practical value $\sigma^{\star}$ that provides, overall, the highest probabilities of rejection of the null hypothesis.

A first class of alternatives to the null hypothesis of symmetry will follow a general idea proposed by [9] to obtain asymmetric copula models. Starting with a symmetric copula $C$, one of the suggested constructions is to let

$$
\mathcal{K}_{\delta}\left(u_{1}, u_{2}\right)=u_{1}^{\delta} C\left(u_{1}^{1-\delta}, u_{2}\right),
$$

for some $\delta \in[0,1]$. One recovers the symmetric copula $C$ by letting $\delta=0$, while the independence copula occurs when $\delta=1$. Otherwise, $\mathcal{K}_{\delta}$ is generally an asymmetric copula; see [17] for identifiability issues in these models and for composite goodness-of-fit tests. When $C$ belongs to the Clayton, Gumbel and Normal classes of copulas, this construction yields what will be referred to the asymmetric Khoudraji-Clayton, KhoudrajiGumbel and Khoudraji-Normal copulas. Instead of the Khoudraji version of the chi-square copula, one will consider a special case of the general construction by considering the dependence structure $C_{a, \theta}^{X}$ of $\left(\left(Z_{1}+\right.\right.$ $\left.a)^{2}, Z_{2}^{2}\right)$, where $\left(Z_{1}, Z_{2}\right)$ is standard normal with correlation $\theta$. Scatterplots of $n=5000$ simulated pairs from the Khoudraji copulas when $\delta=.5$ and $\tau_{C} \in\{.6, .7, .8\}$ are found in Figure 1, as well as for the asymmetric chi-square copula when $a \in\{.5, .75,1\}$ and $\theta=.9$.
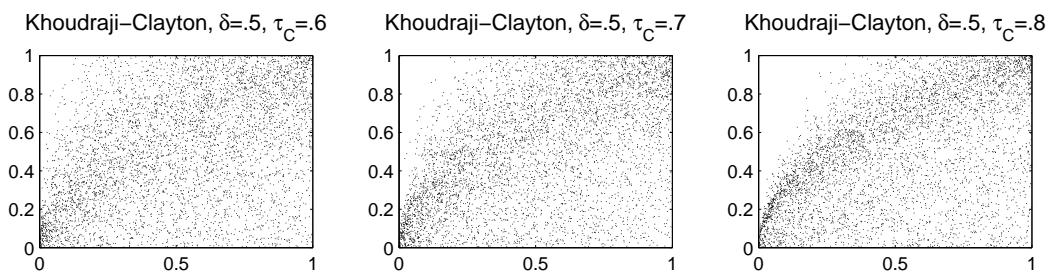

Khoudraji-Gumbel, $\delta=.5, \tau_{C}=.6$

Khoudraji-Gumbel, $\delta=.5, \tau_{\mathrm{C}}=.7$

Khoudraji-Gumbel, $\delta=.5, \tau_{\mathrm{C}}=.8$
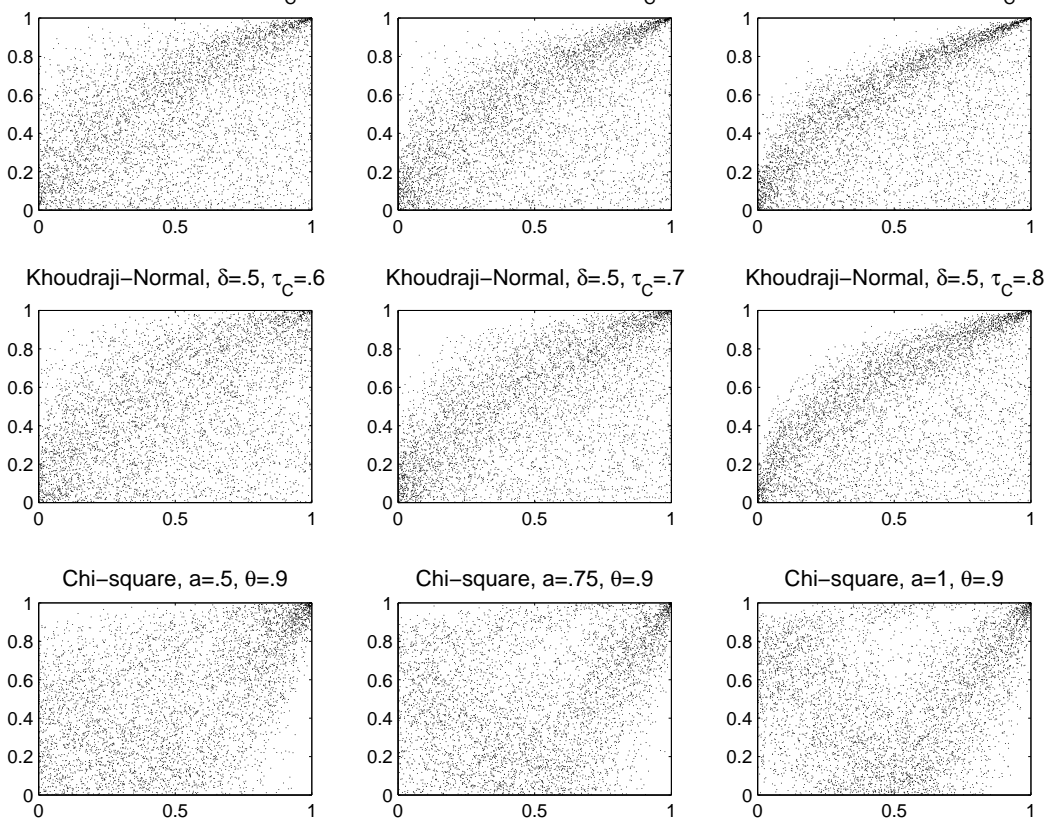

Figure 1: Scatterplots of $n=5000$ simulated pairs from the asymmetric Khoudraji-Clayton, Khoudraji-Gumbel, KhoudrajiNormal and chi-square copulas

Table 2 reports the value of the asymmetry index

$$
\gamma_{C}=3 \sup _{(u, v) \in(0,1)}|C(u, v)-C(v, u)|
$$


proposed by [15] for the twelve models of Figure 1. These indices have been numerically approximated on a sufficiently fine grid in order to ensure a 3-digit accuracy. Note that while $\gamma_{C}=0$ for symmetric copulas, it is shown by [15] that $\gamma_{C} \leq 1$ and that this upper bound is sharp.

Table 2: Nelsen's asymmetry index for the Khoudraji-Clayton, Khoudraji-Gumbel, Khoudraji-Normal and chi-square copulas

\begin{tabular}{llr}
\hline Copula & Parameters & $\gamma_{C}$ \\
\hline & $\left(\tau_{C}, \delta\right)=(.6, .5)$ & .107 \\
K.-Clayton & $\left(\tau_{C}, \delta\right)=(.7, .5)$ & .141 \\
& $\left(\tau_{C}, \delta\right)=(.8, .5)$ & .174 \\
K.-Gumbel & $\left(\tau_{C}, \delta\right)=(.6, .5)$ & .122 \\
& $\left(\tau_{C}, \delta\right)=(.7, .5)$ & .152 \\
K.-Normal & $\left(\tau_{C}, \delta\right)=(.8, .5)$ & .184 \\
& $\left(\tau_{C}, \delta\right)=(.6, .5)$ & .108 \\
Chi-square & $\left(\tau_{C}, \delta\right)=(.7, .5)$ & .141 \\
& $\left(\tau_{C}, \delta\right)=(.8, .5)$ & .175 \\
& $(a, \theta)=(.50, .9)$ & .056 \\
& $(a, \theta)=(.75, .9)$ & .114 \\
& $(a, \theta)=(1.0, .9)$ & .187
\end{tabular}

The curves of the estimated power of $S_{n, \sigma}^{\mathrm{N}}$ and $S_{n, \sigma}^{\mathrm{DE}}$ when $n=100$ as a function of $\sigma$ under the twelve models of Table 2 are in Figure 2. A first look convinces that $\sigma=.5$ is not a good choice. Otherwise, any value of $\sigma$ between 1 and 5 corresponds to estimated powers that are very similar among the twelve models that have been considered. Hence, in the sequel, $\sigma^{\star}=3$ will be used as a universally good choice for the smoothing parameter. Note that under the three Khoudraji-type models, the tests' performances are very similar for a given value of $\tau_{C}$. This behavior can easily be explained by the fact that these dependence structures are almost identical with respect to the kind of asymmetry that they generate, as can be noticed from Figure 1 as well as from the values of $\gamma_{C}$ reported in Table 2.

\subsection{Power comparison with a test of [5]}

The power of $S_{n, \sigma^{\star}}^{\mathrm{N}}$ and $S_{n, \sigma^{\star}}^{\mathrm{DE}}$ when $\sigma^{\star}=3$ will now be compared to a Cramér-von Mises test statistic for copula symmetry suggested by [5], namely

$$
\begin{aligned}
S_{n}^{\mathrm{CvM}} & =n \int_{[0,1]^{2}}\left\{C_{n}\left(u_{1}, u_{2}\right)-C_{n}\left(u_{2}, u_{1}\right)\right\}^{2} \mathrm{~d} C_{n}\left(u_{1}, u_{2}\right) \\
& =\sum_{k=1}^{n}\left\{C_{n}\left(\widehat{U}_{k 1}, \widehat{U}_{k 2}\right)-C_{n}\left(\widehat{U}_{k 2}, \widehat{U}_{k 1}\right)\right\}^{2}
\end{aligned}
$$

where $C_{n}=(1 / n) \sum_{j=1}^{n} \mathbb{I}\left(\widehat{U}_{j 1} \leq u_{1}, \widehat{U}_{j 2} \leq u_{2}\right)$ is the empirical copula. Two other statistics based on $C_{n}$ were also introduced by [5], but they were generally much less powerful than $S_{n}^{\text {CvM }}$. Full details on the computation of $S_{n}^{\mathrm{CvM}}$ and its multiplier bootstrap versions are provided in Appendix B; in particular, easily implementable versions of the multiplier replicates are provided. 

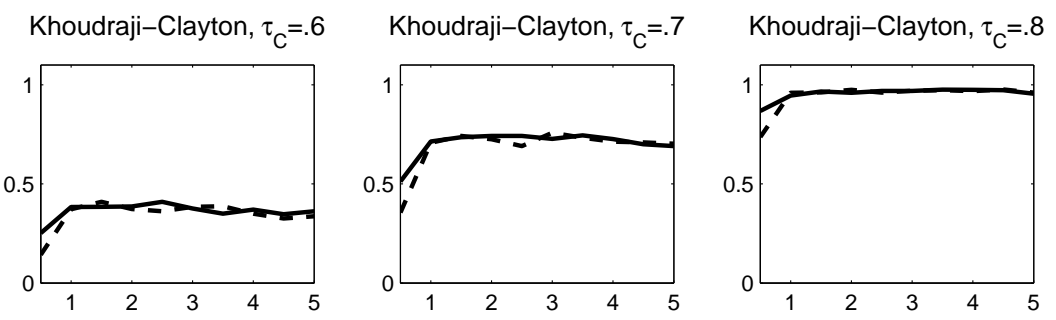

Khoudraji-Gumbel, $\tau_{\mathrm{C}}=.6$
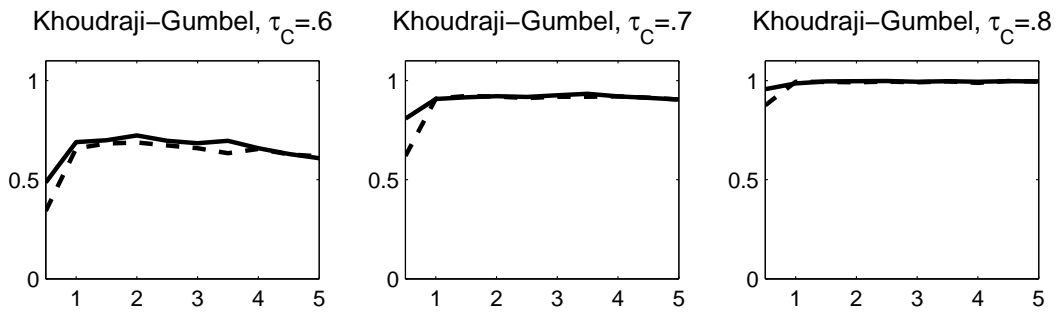

Khoudraji-Normal, $\tau_{\mathrm{C}}=.6$
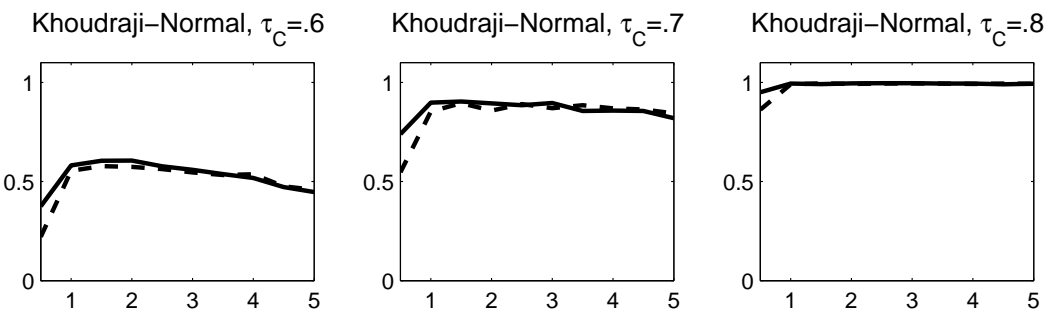

Asymmetric chi-square, $a=.5$ Asymmetric chi-square, $a=.75$ Asymmetric chi-square, $a=1$
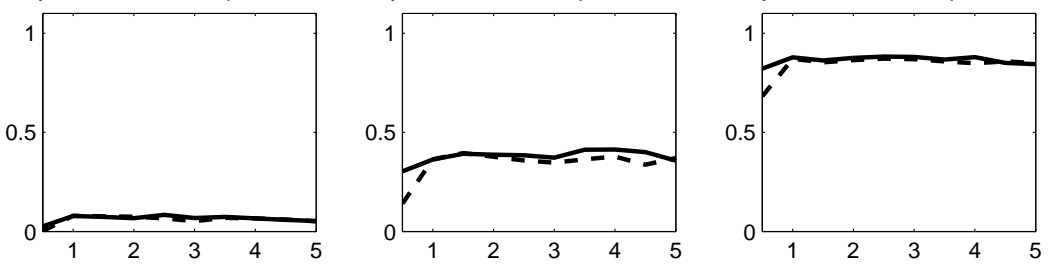

Figure 2: Probabilities of rejection, as estimated from 1000 replicates, of the tests of bivariate copula symmetry based on the characteristic function statistics $S_{n, \sigma}^{N}$ (line) and $S_{n, \sigma}^{D E}$ (dashed line) as a function of $\sigma \in\{.5,1, \ldots, 5\}$, when $n=100$ for the twelve asymmetric copula models of Figure 1

Note that the multiplier method for empirical copulas requires the nonparametric estimation of the partial derivatives of the copula (see Equation (12)), which itself requires the choice of a smoothing parameter that was set to $\ell_{n}=1 / \sqrt{n}$ by [5]. The results comparing $S_{n, \sigma^{*}}^{\mathrm{N}}$ and $S_{n, \sigma^{*}}^{\mathrm{DE}}$ with $S_{n}^{\mathrm{CvM}}$ are in Table 3, where the same twelve asymmetric models as those considered in Figures $1-2$ have been used.

First of all, as expected from the tests' consistency, the power of the three statistics increases as the sample size increases. The power also increases with the level of dependence, a consequence of the fact that the level of asymmetric of the models under consideration increases as a function of $\tau_{C}$. Among the Khoudraji-type models, the asymmetric Gumbel copula is the most easily detected departure from $\mathbb{H}_{0}$, as one could expect from Figure 1. The asymmetry provided by the chi-square copula is well detected when $a \in\{.75,1\}$, but hardly detected when $a=.5$; if one looks at Figure 1, this latter case corresponds to a dependence structure that is close to symmetry (indeed, $\gamma_{C}=.056$ is small for this model). Overall, $S_{n, \sigma^{*}}^{\mathrm{N}}$ is systematically slightly more powerful than $S_{n, \sigma^{*}}^{\mathrm{DE}}$, except under the Khoudraji-Clayton copula when $\tau_{C}=.6$ and $n \in\{175,250\}$. Interestingly, the two characteristic function statistics $S_{n, \sigma^{*}}^{\mathrm{N}}$ and $S_{n, \sigma^{*}}^{\mathrm{DE}}$ are significantly more powerful than $S_{n}^{\mathrm{CvM}}$ under all the considered scenarios. 


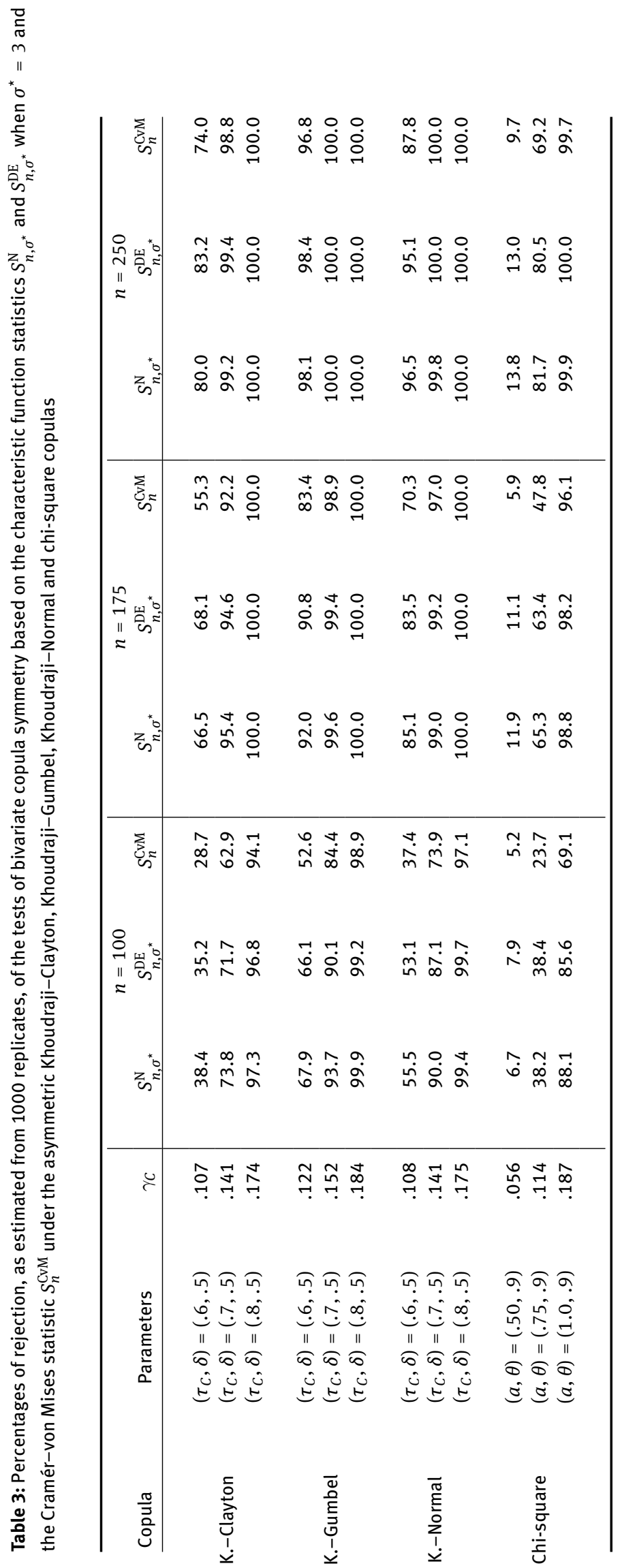




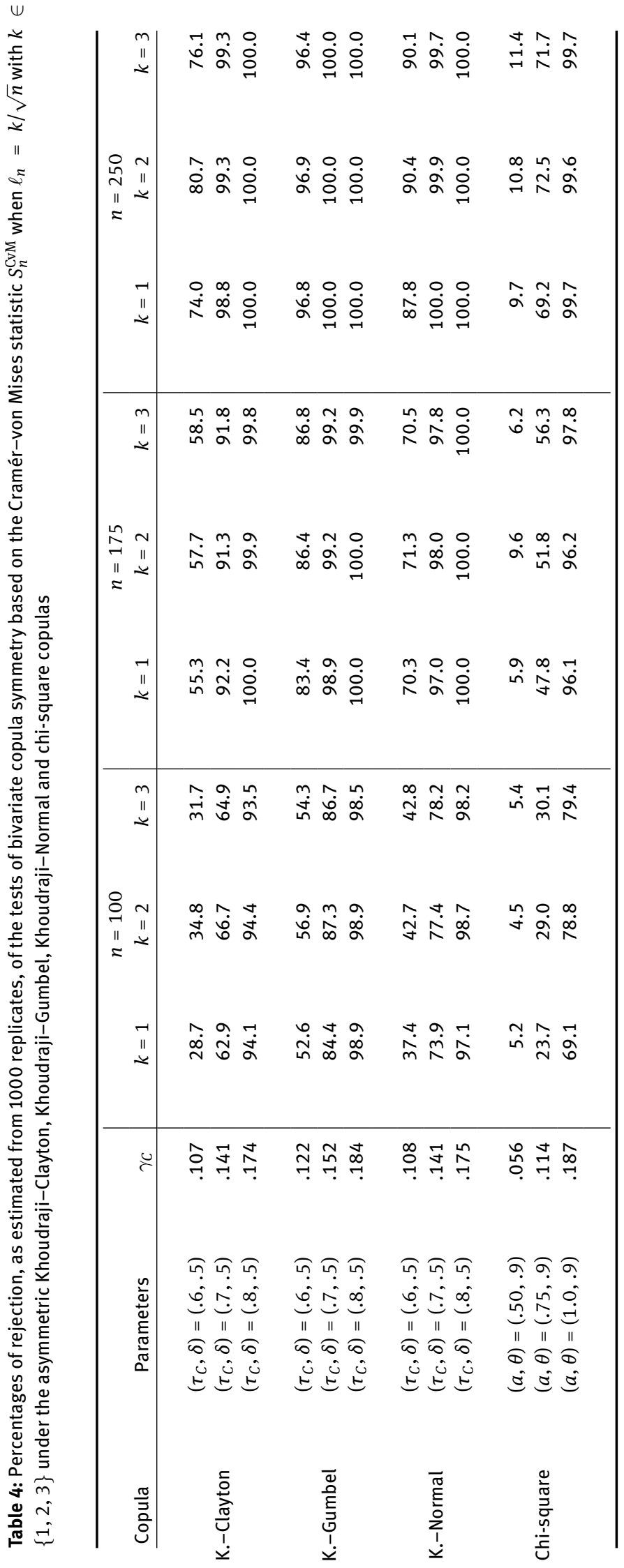


For their tests of radial symmetry for bivariate copulas, [6] observed that the choice of $\ell_{n}$ entering in the estimation of the partial derivatives may have a significant impact on the power. Hence, in order to be as fair as possible when comparing $S_{n, \sigma^{*}}^{\mathrm{N}}$ and $S_{n, \sigma^{*}}^{\mathrm{DE}}$ with $S_{n}^{\mathrm{CvM}}$, additional simulations have been run for three choices of $\ell_{n}$, namely $\ell_{n}=k / \sqrt{n}$ for $k \in\{1,2,3\}$; the results on the estimated power of $S_{n}^{\mathrm{CvM}}$ are found in Table 4. Overall, one can say that while the power is generally higher when $k \in\{2,3\}$ compared to $k=1$, the copula characteristic function tests are still significantly more powerful than $S_{n}^{\mathrm{CvM}}$. Thus, a global conclusion is that one can recommend the use of $S_{n, \sigma^{*}}^{\mathrm{N}}$ and $S_{n, \sigma^{*}}^{\mathrm{DE}}$ for testing bivariate copula symmetry.

\subsection{The Nutrient data set revisited}

The daily intake in calcium (Ca), iron (Fe), protein (Pr), vitamin A (vA) and vitamin C (vC) for $n=747$ women has been collected in 1985 by the United States Department of Agriculture. These data have been analysed by [5] to illustrate their tests of copula symmetry on each of the ten possible pairs of variables. Table 5 reports the p-values, as estimated from $N=10000$ multiplier bootstrap samples, of the tests based on $S_{n, \sigma^{*}}^{\mathrm{N}}$ and $S_{n, \sigma^{*}}^{\mathrm{DE}}$; the p-values of the test based on $S_{n}^{\mathrm{CvM}}$ as reported by [5] are also given. First note that the p-values of the two characteristic-function tests based on $S_{n, \sigma^{*}}^{\mathrm{N}}$ and $S_{n, \sigma^{*}}^{\mathrm{DE}}$ are similar. They, indeed, lead to the same conclusions on the acceptance or rejection of the null hypothesis of symmetry at the $5 \%$ level. Hence, the pairs (Ca, Fe), (Ca,Pr), (Ca,vA), (Fe,vA), $(\mathrm{Fe}, \mathrm{vC})$ and $(\mathrm{Pr}, \mathrm{vA})$ are tested as being significantly asymmetric, while there is not enough evidence to reject $\mathbb{H}_{0}$ for the four other pairs. This is in accordance with the results of the test based on $S_{n}^{\mathrm{CvM}}$.

Table 5: P-values, as estimated from $N=10000$ multiplier samples, of the tests of bivariate copula symmetry based on $S_{n, \sigma^{*}}^{\mathrm{N}}$ and $S_{n, \sigma^{\star}}^{\mathrm{DE}}$ for the ten pairs in the Nutrient data set; $\mathrm{p}$-values of the tests based on $S_{n}^{\mathrm{CvM}}$ as reported by [5] are also given

\begin{tabular}{c|lll}
\hline Pair & $S_{n, \sigma^{*}}^{\mathrm{N}}$ & $S_{n, \sigma^{*}}^{\mathrm{DE}}$ & $S_{n}^{\mathrm{CvM}}$ \\
\hline$(\mathrm{Ca}, \mathrm{Fe})$ & .004 & .005 & .003 \\
$(\mathrm{Ca}, \mathrm{Pr})$ & .000 & .000 & .000 \\
$(\mathrm{Ca}, \mathrm{vA})$ & .000 & .000 & .000 \\
$(\mathrm{Ca}, \mathrm{vC})$ & .221 & .209 & .181 \\
$(\mathrm{Fe}, \mathrm{Pr})$ & .891 & .864 & .402 \\
$(\mathrm{Fe}, \mathrm{vA})$ & .007 & .005 & .002 \\
$(\mathrm{Fe}, \mathrm{vC})$ & .008 & .007 & .004 \\
$(\mathrm{Pr}, \mathrm{VA})$ & .006 & .007 & .004 \\
$(\mathrm{Pr}, \mathrm{vC})$ & .065 & .065 & .136 \\
$(\mathrm{VA}, \mathrm{vC})$ & .832 & .815 & .620 \\
\hline
\end{tabular}

\section{Conclusion}

New tests for the symmetry of bivariate copulas have been proposed around a formulation of the null hypothesis in term of the characteristic function that can be associated to a copula. A general class of test statistics based on nonparametric empirical versions of the copula characteristic function has been defined and their asymptotic distributions under the null hypothesis and under the alternative have been established. The form of the limit distribution under the null hypothesis has then been exploited to provide suitably adapted multiplier versions for the computation of valid $p$-values. The simulation results that are reported are quite convincing on the superiority of the new tests over the procedures of [5] based on the empirical copula. 
Generally speaking, inference methods based on the characteristic function are widely recognized as competing approaches to those adopting a distribution-oriented philosophy. However, the use of the characteristic function is just starting to emerge in the context of copula inference. The asymptotic behavior of the test statistics introduced in this work is typical of weighted $L_{2}$ functionals computed from a rank-based version of the copula characteristic function. In the light of the simulation results presented in this work as well as those in [2] and [1], this approach deserves to be considered as a viable alternative to methods based on the empirical copula.

In future investigations, it would be possible to extend the proposed tests of bivariate copula symmetry to the general $d$-dimensional case, hence providing competing methods to those of [7]. Also, allowing for weak dependence in the data, e.g. $\alpha$-mixing, would be a significant step further; this could be done by adapting results of [12] on the serial multiplier method for standard (no ranks) V-statistics under weakly-dependent data. Finally, being able to choose an optimal weight function and smoothing parameter with a formal criterion would certainly be of interest.

Acknowledgement: Thanks are due to Pierre Lafaye de Micheaux for very constructive comments on an earlier version of the paper. This research was supported in part by individual grants provided to Taoufik Bouezmarni and Jean-François Quessy by the Natural Sciences and Engineering Research Council of Canada and by the Fonds de Recherche du Québec - Nature et Technologies. Jean-François Quessy also acknowledges financial support from a team grant of the Canadian Statistical Science Institute (CANSSI).

\section{A Proofs of the main results}

\section{A.1 Proof of Lemma 2.1}

Using the definition of $\mathcal{L}_{\mathbf{t}}$ in (2), one can write

$$
\Psi_{n}\left(t_{1}, t_{2}\right)-\Psi_{n}\left(t_{2}, t_{1}\right)=\frac{1}{n} \sum_{j=1}^{n} \mathcal{L}_{\mathbf{t}}\left(\widehat{\mathbf{U}}_{j}\right) .
$$

Since the complex conjugate of $\mathcal{L}_{\mathbf{t}}$ is $\mathcal{L}_{-\mathbf{t}}$, one then has

$$
\begin{aligned}
S_{n, \omega} & =\int_{\mathbb{R}^{2}}\left|\frac{1}{n} \sum_{j=1}^{n} \mathcal{L}_{\mathbf{t}}\left(\widehat{\mathbf{U}}_{j}\right)\right|^{2} \omega(\mathbf{t}) \mathrm{d} \mathbf{t} \\
& =\int_{\mathbb{R}^{2}}\left(\frac{1}{n} \sum_{j=1}^{n} \mathcal{L}_{\mathbf{t}}\left(\widehat{\mathbf{U}}_{j}\right)\right)\left(\frac{1}{n} \sum_{k=1}^{n} \mathcal{L}_{-\mathbf{t}}\left(\widehat{\mathbf{U}}_{k}\right)\right) \omega(\mathbf{t}) \mathrm{d} \mathbf{t} \\
& =\frac{1}{n^{2}} \sum_{j, k=1}^{n} \int_{\mathbb{R}^{2}} \mathcal{L}_{\mathbf{t}}\left(\widehat{\mathbf{U}}_{j}\right) \mathcal{L}_{-\mathbf{t}}\left(\widehat{\mathbf{U}}_{k}\right) \omega(\mathbf{t}) \mathrm{d} \mathbf{t} \\
& =\frac{1}{n^{2}} \sum_{j, k=1}^{n} B_{\omega}\left(\widehat{\mathbf{U}}_{j}, \widehat{\mathbf{U}}_{k}\right),
\end{aligned}
$$

where

$$
B_{\omega}(\mathbf{a}, \mathbf{b})=\int_{\mathbb{R}^{2}} \mathcal{L}_{\mathbf{t}}(\mathbf{a}) \mathcal{L}_{-\mathbf{t}}(\mathbf{b}) \omega(\mathbf{t}) \mathrm{d} \mathbf{t} .
$$


Next, one obtains from the definition of $\mathcal{L}_{\mathbf{t}}$ in (2) and some algebra that

$$
\begin{aligned}
\mathcal{L}_{\mathbf{t}}(\mathbf{a}) \mathcal{L}_{-\mathbf{t}}(\mathbf{b})= & \left\{\cos \left(t_{1} a_{1}+t_{2} a_{2}\right)-\cos \left(t_{2} a_{1}+t_{1} a_{2}\right)\right\}\left\{\cos \left(t_{1} b_{1}+t_{2} b_{2}\right)-\cos \left(t_{2} b_{1}+t_{1} b_{2}\right)\right\} \\
& +\left\{\sin \left(t_{1} a_{1}+t_{2} a_{2}\right)-\sin \left(t_{2} a_{1}+t_{1} a_{2}\right)\right\}\left\{\sin \left(t_{1} b_{1}+t_{2} b_{2}\right)-\sin \left(t_{2} b_{1}+t_{1} b_{2}\right)\right\} \\
= & \cos \left\{t_{1}\left(a_{1}-b_{1}\right)+t_{2}\left(a_{2}-b_{2}\right)\right\} \\
& -\cos \left\{t_{1}\left(a_{1}-b_{2}\right)+t_{2}\left(a_{2}-b_{1}\right)\right\} \\
& -\cos \left\{t_{1}\left(a_{2}-b_{1}\right)+t_{2}\left(a_{1}-b_{2}\right)\right\} \\
& +\cos \left\{t_{1}\left(a_{2}-b_{2}\right)+t_{2}\left(a_{1}-b_{1}\right)\right\},
\end{aligned}
$$

where the last equality is a consequence of the trigonometric identity

$$
\cos (x) \cos (y)+\sin (x) \sin (y)=\cos (x-y) .
$$

Using the fact that $\omega$ is symmetric, one finally computes

$$
\begin{aligned}
B_{\omega}(\mathbf{a}, \mathbf{b})= & \int_{\mathbb{R}^{2}} \mathcal{L}_{\mathbf{t}}(\mathbf{a}) \mathcal{L}_{-\mathbf{t}}(\mathbf{b}) \omega(\mathbf{t}) \mathrm{d} \mathbf{t} \\
= & 2 \int_{\mathbb{R}^{2}} \cos \left\{t_{1}\left(a_{1}-b_{1}\right)+t_{2}\left(a_{2}-b_{2}\right)\right\} \omega\left(t_{1}, t_{2}\right) \mathrm{d} t_{1} \mathrm{~d} t_{2} \\
& -2 \int_{\mathbb{R}^{2}} \cos \left\{t_{1}\left(a_{1}-b_{2}\right)+t_{2}\left(a_{2}-b_{1}\right)\right\} \omega\left(t_{1}, t_{2}\right) \mathrm{d} t_{1} \mathrm{~d} t_{2} \\
= & 2 \widetilde{B}_{\omega}\left(a_{1}-b_{1}, a_{2}-b_{2}\right)-2 \widetilde{B}_{\omega}\left(a_{2}-b_{1}, a_{1}-b_{2}\right) .
\end{aligned}
$$

\section{A.2 Proof of Proposition 2.2}

One first starts with a lemma whose proof is postponed to subsection A.5.

Lemma A.1. Let $\left(X_{11}, X_{12}\right), \ldots,\left(X_{n 1}, X_{n 2}\right)$ be i.i.d. from a bivariate distribution function with continuous marginal distributions $F_{1}$ and $F_{2}$ and copula $C$. If $\omega: \mathbb{R}^{2} \rightarrow \mathbb{R}$ is a non-negative and integrable function that satisfies $\int_{\mathbb{R}^{2}}\left(t_{1}+t_{2}\right)^{4} \omega\left(t_{1}, t_{2}\right) \mathrm{d} t_{1} \mathrm{~d} t_{2}<\infty$, then

$$
S_{n, \omega}=\frac{1}{n^{4}} \int_{\mathbb{R}^{2}}\left|\sum_{j, k=1}^{n} \Omega_{\mathbf{t}}\left(\mathbf{U}_{j}, \mathbf{U}_{k}\right)\right|^{2} \omega(\mathbf{t}) \mathrm{d} \mathbf{t}+o_{\mathbb{P}}(1 / n) .
$$

Since the complex conjugate of $\mathcal{L}_{\mathbf{t}}$ is $\mathcal{L}_{-\mathbf{t}}$, one has $\overline{\mathcal{L}_{\mathbf{t}}^{[1]}}=\mathcal{L}_{-\mathbf{t}}^{[1]}$ and $\overline{\mathcal{L}_{\mathbf{t}}^{[2]}}=\mathcal{L}_{-\mathbf{t}}^{[2]}$; this entails $\overline{\Omega_{\mathbf{t}}}=\Omega_{-\mathbf{t}}$. As a consequence, for $\Phi_{\omega}$ defined in (3),

$$
\begin{aligned}
\int_{\mathbb{R}^{2}}\left|\sum_{j, k=1}^{n} \Omega_{\mathbf{t}}\left(\mathbf{U}_{j}, \mathbf{U}_{k}\right)\right|^{2} \omega(\mathbf{t}) \mathrm{d} \mathbf{t} & =\sum_{j, k, j^{\prime}, k^{\prime}=1}^{n} \int_{\mathbb{R}^{2}} \Omega_{\mathbf{t}}\left(\mathbf{U}_{j}, \mathbf{U}_{k}\right) \Omega_{-\mathbf{t}}\left(\mathbf{U}_{j^{\prime}}, \mathbf{U}_{k^{\prime}}\right) \omega(\mathbf{t}) \mathrm{d} \mathbf{t} \\
& =\sum_{j, k, j^{\prime}, k^{\prime}=1}^{n} \Phi_{\omega}\left(\mathbf{U}_{j}, \mathbf{U}_{k}, \mathbf{U}_{j^{\prime}}, \mathbf{U}_{k^{\prime}}\right) .
\end{aligned}
$$

It follows that

$$
S_{n, \omega}=\frac{1}{n^{4}} \sum_{j, k, j^{\prime}, k^{\prime}=1}^{n} \Phi_{\omega}\left(\mathbf{U}_{j}, \mathbf{U}_{k}, \mathbf{U}_{j^{\prime}}, \mathbf{U}_{k^{\prime}}\right)+o_{\mathbb{P}}(1 / n) .
$$

Note that the fact that $\left|\mathcal{L}_{\mathbf{t}}^{[\ell]}(\mathbf{u})\right| \leq\left|t_{\ell}\right|$ and the assumption $\int_{\mathbb{R}^{2}}\left(t_{1}+t_{2}\right)^{4} \omega(\mathbf{t}) \mathrm{d} \mathbf{t}<\infty$ entail the existence of the integral $\int_{\mathbb{R}^{2}} \Omega_{\mathbf{t}}\left(\mathbf{U}_{j}, \mathbf{U}_{k}\right) \Omega_{-\mathbf{t}}\left(\mathbf{U}_{j^{\prime}}, \mathbf{U}_{k^{\prime}}\right) \omega(\mathbf{t}) \mathrm{d} \mathbf{t}$. 


\section{A.3 Proof of Proposition 2.3}

From the representation of a V-statistic as a sum of U-statistics (see for instance Theorem 1, p. 183 of [11]), one deduces that

$$
W_{n, \omega}=W_{n 1, \omega}+\frac{1}{n} W_{n 2, \omega}+o_{\mathbb{P}}(1 / n),
$$

where $W_{n 1, \omega}$ is the U-statistic of degree four with kernel $\Phi_{\omega}$ and $W_{n 2, \omega}$ is the U-statistic of degree three with kernel

$$
\Phi_{2, \omega}\left(\mathbf{u}_{1}, \mathbf{u}_{2}, \mathbf{u}_{3}\right)=2\left\{\Phi_{\omega}\left(\mathbf{u}_{1}, \mathbf{u}_{1}, \mathbf{u}_{2}, \mathbf{u}_{3}\right)+\Phi_{\omega}\left(\mathbf{u}_{2}, \mathbf{u}_{2}, \mathbf{u}_{1}, \mathbf{u}_{3}\right)+\Phi_{\omega}\left(\mathbf{u}_{3}, \mathbf{u}_{3}, \mathbf{u}_{1}, \mathbf{u}_{2}\right)\right\} .
$$

Because $C$ is symmetric, one has for $\mathbf{U}_{1}, \mathbf{U}_{2}, \mathbf{U}_{3}$ i.i.d. $C$ that $\mathrm{E}\left\{\widetilde{\Omega}_{\mathbf{t}}\left(\mathbf{U}_{1}, \mathbf{U}_{2}\right)\right\}=\mathrm{E}\left\{\widetilde{\Omega}_{\mathbf{t}}\left(\mathbf{U}_{2}, \mathbf{U}_{1}\right)\right\}=0$, and then $\mathrm{E}\left\{\Omega_{\mathbf{t}}\left(\mathbf{U}_{1}, \mathbf{U}_{2}\right)\right\}=0$. From the definition of $\Phi_{\omega}$, it can then be shown easily that $\mathrm{E}\left\{\Phi_{\omega}\left(\mathbf{u}_{1}, \mathbf{U}_{2}, \mathbf{U}_{3}, \mathbf{U}_{4}\right)\right\}=0$, i.e. the kernel $\Phi_{\omega}$ is first-order degenerate. From Corollary 1, p. 83 in [11], one deduces that $W_{n 1, \omega}$ is asymptotically equivalent to a U-statistic with kernel

$$
\begin{aligned}
Y_{C, \omega}\left(\mathbf{u}_{1}, \mathbf{u}_{2}\right)= & 6 \mathrm{E}\left\{\Phi_{\omega}\left(\mathbf{u}_{1}, \mathbf{u}_{2}, \mathbf{U}_{1}, \mathbf{U}_{2}\right)\right\} \\
= & 2 \int_{\mathbb{R}^{2}} \Omega_{\mathbf{t}}\left(\mathbf{u}_{1}, \mathbf{u}_{2}\right) \mathrm{E}\left\{\Omega_{-\mathbf{t}}\left(\mathbf{U}_{1}, \mathbf{U}_{2}\right)\right\} \omega(\mathbf{t}) \mathrm{d} \mathbf{t} \\
& +2 \int_{\mathbb{R}^{2}} \mathrm{E}\left\{\Omega_{\mathbf{t}}\left(\mathbf{u}_{1}, \mathbf{U}_{1}\right)\right\} \mathrm{E}\left\{\Omega_{-\mathbf{t}}\left(\mathbf{u}_{2}, \mathbf{U}_{2}\right)\right\} \omega(\mathbf{t}) \mathrm{d} \mathbf{t} \\
& +2 \int_{\mathbb{R}^{2}} \mathrm{E}\left\{\Omega_{\mathbf{t}}\left(\mathbf{u}_{1}, \mathbf{U}_{2}\right)\right\} \mathrm{E}\left\{\Omega_{-\mathbf{t}}\left(\mathbf{u}_{2}, \mathbf{U}_{1}\right)\right\} \omega(\mathbf{t}) \mathrm{d} \mathbf{t} \\
= & 4 \int_{\mathbb{R}^{2}} \mathrm{E}\left\{\Omega_{\mathbf{t}}\left(\mathbf{u}_{1}, \mathbf{U}_{1}\right)\right\} \mathrm{E}\left\{\Omega_{-\mathbf{t}}\left(\mathbf{u}_{2}, \mathbf{U}_{2}\right)\right\} \omega(\mathbf{t}) \mathrm{d} \mathbf{t} \\
= & \int_{\mathbb{R}^{2}} \Lambda_{C, \mathbf{t}}\left(\mathbf{u}_{1}\right) \Lambda_{C,-\mathbf{t}}\left(\mathbf{u}_{2}\right) \omega(\mathbf{t}) \mathrm{d} \mathbf{t},
\end{aligned}
$$

where $\Lambda_{C, \mathbf{t}}(\mathbf{u})=2 \mathrm{E}\left\{\Omega_{\mathbf{t}}(\mathbf{u}, \mathbf{U})\right\}$. Because $\mathcal{L}_{\mathbf{t}}\left(u_{1}, u_{2}\right)=-\mathcal{L}_{\mathbf{t}}\left(u_{2}, u_{1}\right)$, one can show easily that $\mathcal{L}_{\mathbf{t}}^{[2]}\left(u_{1}, u_{2}\right)=$ $-\mathcal{L}_{\mathbf{t}}^{[1]}\left(u_{2}, u_{1}\right)$. Then, for $\mathbf{U}=\left(U_{1}, U_{2}\right) \sim C$,

$$
\mathrm{E}\left\{U_{2} \mathcal{L}_{\mathbf{t}}^{[2]}(\mathbf{U})\right\}=-\mathrm{E}\left\{U_{2} \mathcal{L}_{\mathbf{t}}^{[1]}\left(U_{2}, U_{1}\right)\right\}=-\mathrm{E}\left\{U_{1} \mathcal{L}_{\mathbf{t}}^{[1]}(\mathbf{U})\right\} .
$$

Then, from a simple calculation,

$$
\Lambda_{C, \mathbf{t}}(\mathbf{u})=\mathcal{L}_{\mathbf{t}}(\mathbf{u})+\mathrm{E}\left\{\mathbb{I}\left(U_{1} \geq u_{1}\right) \mathcal{L}_{\mathbf{t}}^{[1]}(\mathbf{U})+\mathbb{I}\left(U_{2} \geq u_{2}\right) \mathcal{L}_{\mathbf{t}}^{[2]}(\mathbf{U})\right\} .
$$

On the other side, invoking for instance Theorem 3, p. 122 of [11], $W_{n 2, \omega}$ converges in probability to

$$
\mathrm{E}\left\{\Phi_{2, \omega}\left(\mathbf{U}_{1}, \mathbf{U}_{2}, \mathbf{U}_{3}\right)\right\}=6 \mathrm{E}\left\{\Phi_{\omega}\left(\mathbf{U}_{1}, \mathbf{U}_{1}, \mathbf{U}_{2}, \mathbf{U}_{3}\right)\right\}=\mathrm{E}\left\{\Psi_{C, \omega}\left(\mathbf{U}_{1}, \mathbf{U}_{1}\right)\right\} .
$$

Putting this together allows to write

$$
\begin{aligned}
W_{n, \omega} & =\frac{1}{n^{2}} \sum_{j=1}^{n} Y_{C, \omega}\left(\mathbf{U}_{j}, \mathbf{U}_{j}\right)+\left(\begin{array}{l}
n \\
2
\end{array}\right)^{-1} \sum_{j<k}^{n} Y_{C, \omega}\left(\mathbf{U}_{j}, \mathbf{U}_{k}\right)+o_{\mathbb{P}}(1 / n) \\
& =\frac{1}{n^{2}} \sum_{j, k=1}^{n} Y_{C, \omega}\left(\mathbf{U}_{j}, \mathbf{U}_{k}\right)+o_{\mathbb{P}}(1 / n) .
\end{aligned}
$$

Finally, since $S_{n, \omega}=W_{n, \omega}+o_{\mathbb{P}}(1 / n)$, it follows that

$$
S_{n, \omega}=\frac{1}{n^{2}} \sum_{j, k=1}^{n} Y_{C, \omega}\left(\mathbf{U}_{j}, \mathbf{U}_{k}\right)+o_{\mathbb{P}}(1 / n) .
$$

Again from Corollary 1, p. 83 of [11] and using Slutsky's lemma, one concludes that $n S_{n, \omega}$ converges in distribution to $\mathbb{S}_{C, \omega}$. 


\section{A.4 Proof of Proposition 2.4}

For $\mathbf{U}_{1}, \mathbf{U}_{2}, \mathbf{U}_{3}, \mathbf{U}_{4}$ i.i.d. $C, \mathrm{E}\left\{\Omega_{\mathbf{t}}\left(\mathbf{U}_{1}, \mathbf{U}_{2}\right)\right\}=\Psi_{C}\left(t_{1}, t_{2}\right)-\Psi_{C}\left(t_{2}, t_{1}\right)$, so that

$$
\begin{aligned}
\Phi_{\omega}^{(1)}\left(\mathbf{u}_{1}\right) & =\mathrm{E}\left\{\Phi_{\omega}\left(\mathbf{u}_{1}, \mathbf{U}_{2}, \mathbf{U}_{3}, \mathbf{U}_{4}\right)\right\} \\
& =\int_{\mathbb{R}^{2}} \mathrm{E}\left\{\Omega_{\mathbf{t}}\left(\mathbf{u}_{1}, \mathbf{U}_{2}\right)\right\}\left\{\Psi_{C}\left(-t_{1},-t_{2}\right)-\Psi_{C}\left(-t_{2},-t_{1}\right)\right\} \omega(\mathbf{t}) \mathrm{d} \mathbf{t} .
\end{aligned}
$$

Since $\mathrm{E}\left\{\Omega_{\mathbf{t}}\left(\mathbf{u}_{1}, \mathbf{U}_{2}\right)\right\} \neq 0$, as well as $\Psi_{C}\left(t_{1}, t_{2}\right)-\Psi_{C}\left(t_{2}, t_{1}\right) \neq 0$ when $C$ is asymmetric, the fact that $\omega$ is strictly positive (except maybe on a subset of $\mathbb{R}^{2}$ with null Lebesgue measure), $\Phi_{\omega}^{(1)}\left(\mathbf{u}_{1}\right) \neq 0$. In other words, the kernel $\Phi_{\omega}$ is non-degenerate. Hence, because

$$
\begin{aligned}
\mathrm{E}\left\{\Phi_{\omega}\left(\mathbf{U}_{1}, \mathbf{U}_{2}, \mathbf{U}_{3}, \mathbf{U}_{4}\right)\right\} & =\int_{\mathbb{R}^{2}}\left\{\Psi_{C}\left(t_{1}, t_{2}\right)-\Psi_{C}\left(t_{2}, t_{1}\right)\right\}\left\{\Psi_{C}\left(-t_{1},-t_{2}\right)-\Psi_{C}\left(-t_{2},-t_{1}\right)\right\} \omega(\mathbf{t}) \mathrm{d} \mathbf{t} \\
& =\int_{\mathbb{R}^{2}}\left|\Psi_{C}\left(t_{1}, t_{2}\right)-\Psi_{C}\left(t_{2}, t_{1}\right)\right|^{2} \omega(\mathbf{t}) \mathrm{d} \mathbf{t} \\
& =\mu_{C, \omega},
\end{aligned}
$$

classical arguments in the theory of U-statistics (see Theorem 1, p. 76 of [11], for instance) entail that $W_{n 1, \omega}$ in (10) is such that $\sqrt{n}\left(W_{n 1, \omega}-\mu_{C, \omega}\right)$ is asymptotically normal with mean zero and variance $\sigma_{C, \omega}^{2}=$ $16 \operatorname{Var}\left\{\Phi_{\omega}^{(1)}\left(\mathbf{U}_{1}\right)\right\}$. The proof is complete upon noting that $\sqrt{n}\left(W_{n, \omega}-\mu_{C, \omega}\right)=\sqrt{n}\left(W_{n 1, \omega}-\mu_{C, \omega}\right)+o_{\mathbb{P}}(1 / \sqrt{n})$ and applying Slutsky's Lemma.

\section{A.5 Proof of Lemma A.1}

From the definition of $\mathcal{L}_{\mathbf{t}}$ in (2), one can write the test statistic as

$$
S_{n, \omega}=\frac{1}{n^{2}} \int_{\mathbb{R}^{2}}\left|\sum_{j=1}^{n} \mathcal{L}_{\mathbf{t}}\left(\widehat{\mathbf{U}}_{j}\right)\right|^{2} \omega(\mathbf{t}) \mathrm{d} \mathbf{t} .
$$

By invoking the mean-value Theorem, one has

$$
\begin{aligned}
\sum_{j=1}^{n} \mathcal{L}_{\mathbf{t}}\left(\widehat{\mathbf{U}}_{j}\right)= & \sum_{j=1}^{n}\left\{\mathcal{L}_{\mathbf{t}}\left(\mathbf{U}_{j}\right)+\left(\widehat{U}_{j 1}-U_{j 1}\right) \mathcal{L}_{\mathbf{t}}^{[1]}\left(\mathbf{U}_{j}\right)+\left(\widehat{U}_{j 2}-U_{j 2}\right) \mathcal{L}_{\mathbf{t}}^{[2]}\left(\mathbf{U}_{j}\right)\right\} \\
& +\Delta_{n}(\mathbf{t}),
\end{aligned}
$$

where for $\widetilde{\mathbf{U}}_{j}$ between $\widehat{\mathbf{U}}_{j}$ and $\mathbf{U}_{j}$, and $\nabla^{2} \mathcal{L}_{\mathbf{t}}$ being the Hessian matrix of $\mathcal{L}_{\mathbf{t}}$,

$$
\Delta_{n}(\mathbf{t})=\frac{1}{2} \sum_{j=1}^{n}\left(\widehat{\mathbf{U}}_{j}-\mathbf{U}_{j}\right)\left\{\nabla^{2} \mathcal{L}_{\mathbf{t}}\left(\widetilde{\mathbf{U}}_{j}\right)\right\}\left(\widehat{\mathbf{U}}_{j}-\mathbf{U}_{j}\right)^{\top} .
$$

Upon noting that

$$
\widehat{U}_{j 1}=\frac{1}{n+1} \sum_{k=1}^{n} \mathbb{I}\left(U_{k 1} \leq U_{j 1}\right) \quad \text { and } \quad \widehat{U}_{j 2}=\frac{1}{n+1} \sum_{k=1}^{n} \mathbb{I}\left(U_{k 2} \leq U_{j 2}\right) \text {, }
$$

one can then write, in the light of the definition of $\widetilde{\Omega}_{\mathbf{t}}$ and $\Omega_{\mathbf{t}}$,

$$
\sum_{j=1}^{n} \mathcal{L}_{\mathbf{t}}\left(\widehat{\mathbf{U}}_{j}\right)=\frac{1}{n+1} \sum_{j, k=1}^{n} \widetilde{\Omega}_{\mathbf{t}}\left(\mathbf{U}_{j}, \mathbf{U}_{k}\right)+\Delta_{n}(\mathbf{t})=\frac{1}{n+1} \sum_{j, k=1}^{n} \Omega_{\mathbf{t}}\left(\mathbf{U}_{j}, \mathbf{U}_{k}\right)+\Delta_{n}(\mathbf{t}) .
$$


It follows that

$$
S_{n, \omega}=\frac{1}{n^{2}} \int_{\mathbb{R}^{2}}\left|\frac{1}{n+1} \sum_{j, k=1}^{n} \Omega_{\mathbf{t}}\left(\mathbf{U}_{j}, \mathbf{U}_{k}\right)+\Delta_{n}(\mathbf{t})\right|^{2} \omega(\mathbf{t}) \mathrm{d} \mathbf{t} .
$$

Then, using the fact that for any complex numbers $z_{1}$ and $z_{2}$, one has $\left|z_{1}+z_{2}\right|^{2}=\left|z_{1}\right|^{2}+\left|z_{2}\right|^{2}+2 \operatorname{Re}\left(z_{1} \overline{z_{2}}\right)$, it follows that $S_{n, \omega}=W_{n, \omega}+\Delta_{n 1, \omega}+2 \Delta_{n 2, \omega}$, where

$$
\begin{aligned}
& W_{n, \omega}=\frac{1}{n^{2}(n+1)^{2}} \int_{\mathbb{R}^{2}}\left|\sum_{j, k=1}^{n} \Omega_{\mathbf{t}}\left(\mathbf{U}_{j}, \mathbf{U}_{k}\right)\right|^{2} \omega(\mathbf{t}) \mathrm{d} \mathbf{t}, \\
& \Delta_{n 1, \omega}=\frac{1}{n^{2}} \int_{\mathbb{R}^{2}}\left|\Delta_{n}(\mathbf{t})\right|^{2} \omega(\mathbf{t}) \mathrm{d} \mathbf{t}, \\
& \Delta_{n 2, \omega}=\frac{1}{n^{2}(n+1)} \int_{\mathbb{R}^{2}} \operatorname{Re}\left\{\sum_{j, k=1}^{n} \Omega_{\mathbf{t}}\left(\mathbf{U}_{j}, \mathbf{U}_{k}\right) \overline{\Delta_{n}(\mathbf{t})}\right\} \omega(\mathbf{t}) \mathrm{d} \mathbf{t} .
\end{aligned}
$$

It will next be shown that $\Delta_{n 1, \omega}=o_{\mathbb{P}}(1 / n)$. To this end, first write

$$
\Delta_{n 1, \omega}=\frac{1}{n^{2}} \int_{\mathbb{R}^{2}}\left\{\left|\Delta_{n}^{\mathrm{Re}}(\mathbf{t})\right|^{2}+\left|\Delta_{n}^{\mathrm{Im}}(\mathbf{t})\right|^{2}\right\} \omega(\mathbf{t}) \mathrm{d} \mathbf{t},
$$

where $\Delta_{n}^{\mathrm{Re}}$ and $\Delta_{n}^{\mathrm{Im}}$ are the real and imaginary parts, respectively, of $\Delta_{n}$. From straightforward computations, one deduces that for $\mathbf{x}=\left(x_{1}, x_{2}\right)$,

$$
\mathbf{x}\left\{\nabla^{2} \mathcal{L}_{\mathbf{t}}(\mathbf{u})\right\} \mathbf{x}^{\top}=e^{i\left(t_{2} u_{1}+t_{1} u_{2}\right)}\left(t_{2} x_{1}+t_{1} x_{2}\right)^{2}-e^{i\left(t_{1} u_{1}+t_{2} u_{2}\right)}\left(t_{1} x_{1}+t_{2} x_{2}\right)^{2} .
$$

As a consequence,

$$
\left|\operatorname{Re}\left(\mathbf{x}\left\{\nabla^{2} \mathcal{L}_{\mathbf{t}}(\mathbf{u})\right\} \mathbf{x}^{\top}\right)\right| \leq\left(t_{2} x_{1}+t_{1} x_{2}\right)^{2}+\left(t_{1} x_{1}+t_{2} x_{2}\right)^{2} .
$$

It follows from the definition of $\Delta_{n}$ and the above inequality that

$$
\begin{aligned}
\left|\Delta_{n}^{\mathrm{Re}}(\mathbf{t})\right| \leq & \frac{1}{2} \sum_{j=1}^{n}\left|\operatorname{Re}\left\{\left(\widehat{\mathbf{U}}_{j}-\mathbf{U}_{j}\right)\left\{\nabla^{2} \mathcal{L}_{\mathbf{t}}\left(\widehat{\mathbf{U}}_{j}\right)\right\}\left(\widehat{\mathbf{U}}_{j}-\mathbf{U}_{j}\right)^{\top}\right\}\right| \\
\leq & \frac{1}{2} \sum_{j=1}^{n}\left\{\left|t_{2}\left(\widehat{U}_{j 1}-U_{j 1}\right)+t_{1}\left(\widehat{U}_{j 2}-U_{j 2}\right)\right|^{2}\right. \\
& \left.+\left|t_{1}\left(\widehat{U}_{j 1}-U_{j 1}\right)+t_{2}\left(\widehat{U}_{j 2}-U_{j 2}\right)\right|^{2}\right\} .
\end{aligned}
$$

Next, if one defines the re-scaled empirical distribution function

$$
H_{n}\left(x_{1}, x_{2}\right)=\frac{1}{n+1} \sum_{k=1}^{n} \mathbb{I}\left(X_{k 1} \leq x_{1}, X_{k 2} \leq x_{2}\right),
$$

one can write $\widehat{U}_{j 1}-U_{j 1}=\mathbb{H}_{n}\left(X_{j 1}, \infty\right) / \sqrt{n}$ and $\widehat{U}_{j 2}-U_{j 2}=\mathbb{H}_{n}\left(\infty, X_{j 2}\right) / \sqrt{n}$, where $\mathbb{H}_{n}\left(x_{1}, x_{2}\right)=$ $\sqrt{n}\left[H_{n}\left(x_{1}, x_{2}\right)-C\left\{F_{1}\left(x_{1}\right), F_{2}\left(x_{2}\right)\right\}\right]$. It follows that for any $j \in\{1, \ldots, n\}$,

$$
\begin{aligned}
\left|t_{2}\left(\widehat{U}_{j 1}-U_{j 1}\right)+t_{1}\left(\widehat{U}_{j 2}-U_{j 2}\right)\right|^{2} & =\frac{\left|t_{2} \mathbb{H}_{n}\left(X_{j 1}, \infty\right)+t_{1} \mathbb{H}_{n}\left(\infty, X_{j 2}\right)\right|^{2}}{n} \\
& \leq \frac{1}{n}\left(t_{1}+t_{2}\right)^{2} \sup _{\left(x_{1}, x_{2}\right) \in \mathbb{R}^{2}}\left|\mathbb{H}_{n}\left(x_{1}, x_{2}\right)\right|^{2} \\
& =\frac{1}{n}\left(t_{1}+t_{2}\right)^{2}\left\{\sup _{\left(x_{1}, x_{2}\right) \in \mathbb{R}^{2}}\left|\mathbb{H}_{n}\left(x_{1}, x_{2}\right)\right|\right\}^{2} .
\end{aligned}
$$


Hence, one can deduce that

$$
\left|\Delta_{n}^{\mathrm{Re}}(\mathbf{t})\right|^{2} \leq\left(t_{1}+t_{2}\right)^{4}\left\{\sup _{\left(x_{1}, x_{2}\right) \in \mathbb{R}^{2}}\left|\mathbb{H}_{n}\left(x_{1}, x_{2}\right)\right|\right\}^{4} .
$$

One can show that this inequality holds also for $\left|\Delta_{n}^{\operatorname{Im}}(\mathbf{t})\right|^{2}$, and then from (11),

$$
\Delta_{n 1, \omega} \leq \frac{2}{n^{2}}\left\{\sup _{\left(x_{1}, x_{2}\right) \in \mathbb{R}^{2}}\left|\mathbb{H}_{n}\left(x_{1}, x_{2}\right)\right|\right\}^{4} \int_{\mathbb{R}^{2}}\left(t_{1}+t_{2}\right)^{4} \omega(\mathbf{t}) \mathrm{dt} .
$$

From [19], for instance,

$$
\sup _{\left(x_{1}, x_{2}\right) \in \mathbb{R}^{2}}\left|\mathbb{H}_{n}\left(x_{1}, x_{2}\right)\right|=O_{\mathbb{P}}(1) .
$$

Hence, since $\int_{\mathbb{R}^{2}}\left(t_{1}+t_{2}\right)^{4} \omega(\mathbf{t}) \mathrm{d} \mathbf{t}<\infty$, one concludes that $\Delta_{n 1, \omega}=o_{\mathbb{P}}(1 / n)$. Next, because $|\operatorname{Re}(z)| \leq|z|$ for any complex number $z$, one has

$$
\begin{aligned}
\left|\Delta_{n 2, \omega}\right| & =\frac{1}{n^{2}(n+1)}\left|\operatorname{Re}\left\{\int_{\mathbb{R}^{2}} \sum_{j, k=1}^{n} \Omega_{\mathbf{t}}\left(\mathbf{U}_{j}, \mathbf{U}_{k}\right) \overline{\Delta_{n}(\mathbf{t})} \omega(\mathbf{t}) \mathrm{d} \mathbf{t}\right\}\right| \\
& \leq \frac{1}{n^{2}(n+1)}\left|\int_{\mathbb{R}^{2}} \sum_{j, k=1}^{n} \Omega_{\mathbf{t}}\left(\mathbf{U}_{j}, \mathbf{U}_{k}\right) \overline{\Delta_{n}(\mathbf{t})} \omega(\mathbf{t}) \mathrm{d} \mathbf{t}\right| .
\end{aligned}
$$

Now let

$$
f(\mathbf{t})=\frac{1}{n(n+1)} \sum_{j, k=1}^{n} \Omega_{\mathbf{t}}\left(\mathbf{U}_{j}, \mathbf{U}_{k}\right) \sqrt{\omega(\mathbf{t})} \text { and } g(\mathbf{t})=\frac{1}{n} \Delta_{n}(\mathbf{t}) \sqrt{\omega(\mathbf{t})} .
$$

Since for any square integrable complex functions $f$ and $g$, the Cauchy-Schwartz inequality entails

$$
\left|\int_{\mathbb{R}^{2}} f(\mathbf{t}) \overline{g(\mathbf{t})} \mathrm{d} \mathbf{t}\right| \leq\left(\int_{\mathbb{R}^{2}}|f(\mathbf{t})|^{2} \mathrm{~d} \mathbf{t}\right)^{1 / 2}\left(\int_{\mathbb{R}^{2}}|g(\mathbf{t})|^{2} \mathrm{dt}\right)^{1 / 2},
$$

one finally deduces that $\left|\Delta_{n 2, \omega}\right| \leq \sqrt{W_{n, \omega} \Delta_{n 1, \omega}}$. Because $W_{n, \omega}$ is asymptotically equivalent to a V-statistic with bounded kernel (as shown in the proof of Proposition 2.3), $\Delta_{n 2, \omega}=o_{\mathbb{P}}(1 / n)$. It follows that

$$
\begin{aligned}
S_{n, \omega} & =W_{n, \omega}+o_{\mathbb{P}}(1 / n) \\
& =\frac{1}{n^{2}(n+1)^{2}} \int_{\mathbb{R}^{2}}\left|\sum_{j, k=1}^{n} \Omega_{\mathbf{t}}\left(\mathbf{U}_{j}, \mathbf{U}_{k}\right)\right|^{2} \omega(\mathbf{t}) \mathrm{d} \mathbf{t}+o_{\mathbb{P}}(1 / n) \\
& =\frac{1}{n^{4}} \int_{\mathbb{R}^{2}}\left|\sum_{j, k=1}^{n} \Omega_{\mathbf{t}}\left(\mathbf{U}_{j}, \mathbf{U}_{k}\right)\right|^{2} \omega(\mathbf{t}) \mathrm{d} \mathbf{t}+o_{\mathbb{P}}(1 / n) .
\end{aligned}
$$

\section{A.6 Proof of Proposition 3.1}

First note that

$$
\begin{aligned}
\widehat{S}_{n, \omega} & =\int_{\mathbb{R}^{2}}\left|\frac{1}{\sqrt{n}} \sum_{j=1}^{n}\left(\frac{\xi_{j}}{\bar{\xi}}-1\right) \widehat{\Lambda}_{\mathbf{t}}\left(\widehat{\mathbf{U}}_{j}\right)\right|^{2} \omega(\mathbf{t}) \mathrm{d} \mathbf{t} \\
& =\int_{\mathbb{R}^{2}}\left|\frac{1}{\sqrt{n}} \sum_{j=1}^{n}\left(\frac{\xi_{j}}{\bar{\xi}}-1\right) \Lambda_{C, \mathbf{t}}\left(\mathbf{U}_{j}\right) \quad+\frac{1}{\sqrt{n}} \sum_{j=1}^{n}\left(\frac{\xi_{j}}{\bar{\xi}}-1\right)\left\{\widehat{\Lambda}_{\mathbf{t}}\left(\widehat{\mathbf{U}}_{j}\right)-\Lambda_{C, \mathbf{t}}\left(\mathbf{U}_{j}\right)\right\}\right|^{2} \omega(\mathbf{t}) \mathrm{d} \mathbf{t} \\
& =\widetilde{S}_{n, \omega}+\widehat{\Delta}_{n 1, \omega}+2 \widehat{\Delta}_{n 2, \omega},
\end{aligned}
$$


where

$$
\begin{aligned}
\widetilde{S}_{n, \omega} & =\int_{\mathbb{R}^{2}}\left|\frac{1}{\sqrt{n}} \sum_{j=1}^{n}\left(\frac{\xi_{j}}{\bar{\xi}}-1\right) \Lambda_{C, \mathbf{t}}\left(\mathbf{U}_{j}\right)\right|^{2} \omega(\mathbf{t}) \mathrm{d} \mathbf{t} \\
& =\frac{1}{n} \sum_{j, k=1}^{n}\left(\frac{\xi_{j}}{\bar{\xi}}-1\right)\left(\frac{\xi_{k}}{\bar{\xi}}-1\right) Y_{C, \omega}\left(\mathbf{U}_{j}, \mathbf{U}_{k}\right), \\
\widehat{\Delta}_{n 1, \omega} & =\int_{\mathbb{R}^{2}}\left|\frac{1}{\sqrt{n}} \sum_{j=1}^{n}\left(\frac{\xi_{j}}{\bar{\xi}}-1\right)\left\{\widehat{\Lambda}_{\mathbf{t}}\left(\widehat{\mathbf{U}}_{j}\right)-\Lambda_{C, \mathbf{t}}\left(\mathbf{U}_{j}\right)\right\}\right|^{2} \omega(\mathbf{t}) \mathrm{d} \mathbf{t},
\end{aligned}
$$

and from arguments similar as those at the end of the proof of Lemma A.1,

$$
\left|\widehat{\Delta}_{n 2, \omega}\right| \leq \sqrt{\widetilde{S}_{n, \omega} \widehat{\Delta}_{n 1, \omega}} .
$$

Hence, it remains to show that $\widehat{\Delta}_{n 1, \omega}=o_{\mathbb{P}^{\star}}(1)$. To this end, note that since $\bar{\xi} \rightarrow 1$ in probability, it can be shown easily that

$$
\widehat{\Delta}_{n 1, \omega}-\int_{\mathbb{R}^{2}}\left|\frac{1}{\sqrt{n}} \sum_{j=1}^{n}\left(\xi_{j}-1\right)\left\{\widehat{\Lambda}_{\mathbf{t}}\left(\widehat{\mathbf{U}}_{j}\right)-\Lambda_{C, \mathbf{t}}\left(\mathbf{U}_{j}\right)\right\}\right|^{2} \omega(\mathbf{t}) \mathrm{d} \mathbf{t}=o_{\mathbb{P}^{*}}(1) .
$$

Letting $\operatorname{Var}^{\star}(\cdot)$ be the variance conditional on the data, it follows that

$$
\begin{aligned}
V_{n}^{\star}(\mathbf{t}) & =\operatorname{Var}^{*}\left[\frac{1}{\sqrt{n}} \sum_{j=1}^{n}\left(\xi_{j}-1\right)\left\{\widehat{\Lambda}_{\mathbf{t}}\left(\widehat{\mathbf{U}}_{j}\right)-\Lambda_{C, \mathbf{t}}\left(\mathbf{U}_{j}\right)\right\}\right] \\
& =\frac{1}{n} \sum_{j=1}^{n}\left\{\widehat{\Lambda}_{\mathbf{t}}\left(\widehat{\mathbf{U}}_{j}\right)-\Lambda_{C, \mathbf{t}}\left(\mathbf{U}_{j}\right)\right\}^{2} \\
& =\frac{1}{n} \sum_{j=1}^{n}\left\{\mathcal{L}_{\mathbf{t}}\left(\widehat{\mathbf{U}}_{j}\right)-\mathcal{L}_{\mathbf{t}}\left(\mathbf{U}_{j}\right)+\widehat{\mathcal{L}}_{\mathbf{t}, 1}\left(\widehat{\mathbf{U}}_{j}\right)+\widehat{\mathcal{L}}_{\mathbf{t}, 2}\left(\widehat{\mathbf{U}}_{j}\right)\right\}^{2},
\end{aligned}
$$

where for $\ell \in\{1,2\}$,

$$
\widehat{\mathcal{L}}_{\mathbf{t}, \ell}(u)=\frac{1}{n} \sum_{k=1}^{n} \mathbb{I}\left(\widehat{U}_{k \ell} \geq u\right) \mathcal{L}_{\mathbf{t}}^{[\ell]}\left(\widehat{\mathbf{U}}_{k}\right)-\mathrm{E}\left\{\mathbb{I}\left(U_{\ell} \geq u\right) \mathcal{L}_{\mathbf{t}}^{[\ell]}(\mathbf{U})\right\} .
$$

By the mean-value Theorem, one has for $\widetilde{\mathbf{U}}_{j}$ between $\widehat{\mathbf{U}}_{j}$ and $\mathbf{U}_{j}$ that

$$
\mathcal{L}_{\mathbf{t}}\left(\widehat{\mathbf{U}}_{j}\right)-\mathcal{L}_{\mathbf{t}}\left(\mathbf{U}_{j}\right)=\left(\widehat{U}_{j 1}-U_{j 1}\right) \mathcal{L}_{\mathbf{t}}^{[1]}\left(\widetilde{\mathbf{U}}_{j}\right)+\left(\widehat{U}_{j 2}-U_{j 2}\right) \mathcal{L}_{\mathbf{t}}^{[2]}\left(\widetilde{\mathbf{U}}_{j}\right) .
$$

As a consequence,

$$
\begin{aligned}
V_{n}^{\star}(\mathbf{t}) & =\frac{1}{n} \sum_{j=1}^{n}\left\{\left(\widehat{U}_{j 1}-U_{j 1}\right) \mathcal{L}_{\mathbf{t}}^{[1]}\left(\widetilde{\mathbf{U}}_{j}\right)+\left(\widehat{U}_{j 2}-U_{j 2}\right) \mathcal{L}_{\mathbf{t}}^{[2]}\left(\widetilde{\mathbf{U}}_{j}\right)+\widehat{\mathcal{L}}_{\mathbf{t}, 1}\left(\widehat{\mathbf{U}}_{j}\right)+\widehat{\mathcal{L}}_{\mathbf{t}, 2}\left(\widehat{\mathbf{U}}_{j}\right)\right\}^{2} \\
& \leq \max _{1 \leq j \leq n}\left\{\left(\widehat{U}_{j 1}-U_{j 1}\right) \mathcal{L}_{\mathbf{t}}^{[1]}\left(\widetilde{\mathbf{U}}_{j}\right)+\left(\widehat{U}_{j 2}-U_{j 2}\right) \mathcal{L}_{\mathbf{t}}^{[2]}\left(\widetilde{\mathbf{U}}_{j}\right)+\widehat{\mathcal{L}}_{\mathbf{t}, 1}\left(\widehat{\mathbf{U}}_{j}\right)+\widehat{\mathcal{L}}_{\mathbf{t}, 2}\left(\widehat{\mathbf{U}}_{j}\right)\right\}^{2} \\
& \leq 4\left\{V_{n 1}^{\star}(\mathbf{t})+V_{n 2}^{\star}(\mathbf{t})+V_{n 3}^{\star}(\mathbf{t})+V_{n 4}^{\star}(\mathbf{t})\right\},
\end{aligned}
$$

where

$$
\begin{aligned}
& V_{n 1}^{\star}(\mathbf{t})=\max _{1 \leq j \leq n}\left(\widehat{U}_{j 1}-U_{j 1}\right)^{2}\left(\mathcal{L}_{\mathbf{t}}^{[1]}\left(\widetilde{\mathbf{U}}_{j}\right)\right)^{2}, \quad V_{n 2}^{\star}(\mathbf{t})=\max _{1 \leq j \leq n}\left(\widehat{U}_{j 2}-U_{j 2}\right)^{2}\left(\mathcal{L}_{\mathbf{t}}^{[2]}\left(\widetilde{\mathbf{U}}_{j}\right)\right)^{2}, \\
& V_{n 3}^{\star}(\mathbf{t})=\max _{1 \leq j \leq n}\left(\widehat{\mathcal{L}}_{\mathbf{t}, 1}\left(\widehat{\mathbf{U}}_{j}\right)\right)^{2} \quad \text { and } \quad V_{n 4}^{\star}(\mathbf{t})=\max _{1 \leq j \leq n}\left(\widehat{\mathcal{L}}_{\mathbf{t}, 2}\left(\widehat{\mathbf{U}}_{j}\right)\right)^{2} .
\end{aligned}
$$


Because $\left|\mathcal{L}_{\mathbf{t}}^{[1]}(\mathbf{u})\right| \leq\left|t_{1}\right|$ and $\left|\mathcal{L}_{\mathbf{t}}^{[2]}(\mathbf{u})\right| \leq\left|t_{2}\right|$, one has

$$
\begin{aligned}
V_{n 1}^{\star}(\mathbf{t}) & \leq t_{1}^{2} \max _{1 \leq j \leq n}\left(\widehat{U}_{j 1}-U_{j 1}\right)^{2} \\
& =t_{1}^{2} \max _{1 \leq j \leq n}\left(\frac{\mathbb{H}_{n}\left(X_{j 1}, \infty\right)}{\sqrt{n}}\right)^{2} \\
& \leq t_{1}^{2} \sup _{x \in \mathbb{R}}\left(\frac{\mathbb{H}_{n}(x, \infty)}{\sqrt{n}}\right)^{2} \\
& =t_{1}^{2} O_{\mathbb{P}}(1 / n) .
\end{aligned}
$$

Similarly, $V_{n 2}^{\star}(\mathbf{t})=t_{2}^{2} O_{\mathbb{P}}(1 / n)$. Next, let $\mathcal{L}_{\mathbf{t}}^{[11]}(\mathbf{u})=\partial^{2} \mathcal{L}_{\mathbf{t}}(\mathbf{u}) / \partial^{2} u_{1}$ and $\mathcal{L}_{\mathbf{t}}^{[12]}(\mathbf{u})=\partial^{2} \mathcal{L}_{\mathbf{t}}(\mathbf{u}) / \partial u_{1} \partial u_{2}$. Using the fact that $\mathbb{I}\left(\widehat{U}_{k 1} \geq \widehat{U}_{j 1}\right)=\mathbb{I}\left(U_{k 1} \geq U_{j 1}\right)$,

$$
\begin{aligned}
V_{n 3}^{\star}(\mathbf{t})= & \max _{1 \leq j \leq n}\left[\frac{1}{n} \sum_{k=1}^{n} \mathbb{I}\left(U_{k 1} \geq U_{j 1}\right) \mathcal{L}_{\mathbf{t}}^{[1]}\left(\widehat{\mathbf{U}}_{k}\right)-\mathrm{E}\left\{\mathbb{I}\left(U_{1} \geq \widehat{U}_{j 1}\right) \mathcal{L}_{\mathbf{t}}^{[1]}(\mathbf{U})\right\}\right]^{2} \\
= & \max _{1 \leq j \leq n}\left[\frac{1}{n} \sum_{k=1}^{n} \mathbb{I}\left(U_{k 1} \geq U_{j 1}\right) \mathcal{L}_{\mathbf{t}}^{[1]}\left(\mathbf{U}_{k}\right)-\mathrm{E}\left\{\mathbb{I}\left(U_{1} \geq U_{j 1}\right) \mathcal{L}_{\mathbf{t}}^{[1]}(\mathbf{U})\right\}\right. \\
& +\mathbb{I}\left(U_{k 1} \geq U_{j 1}\right)\left\{\left(\widehat{U}_{k 1}-U_{k 1}\right) \mathcal{L}_{\mathbf{t}}^{[11]}\left(\widetilde{\mathbf{U}}_{k}\right)+\left(\widehat{U}_{k 2}-U_{k 2}\right) \mathcal{L}_{\mathbf{t}}^{[12]}\left(\widetilde{\mathbf{U}}_{k}\right)\right\} \\
& \left.+\mathrm{E}\left\{\left(\mathbb{I}\left(U_{1} \geq U_{j 1}\right)-\mathbb{I}\left(U_{1} \geq \widehat{U}_{j 1}\right)\right) \mathcal{L}_{\mathbf{t}}^{[1]}(\mathbf{U})\right\}\right]^{2} \\
\leq & \max _{1 \leq j \leq n}\left[\frac{1}{n} \sum_{k=1}^{n} \mathbb{I}\left(U_{k 1} \geq U_{j 1}\right) \mathcal{L}_{\mathbf{t}}^{[1]}\left(\mathbf{U}_{k}\right)-\mathrm{E}\left\{\mathbb{I}\left(U_{1} \geq U_{j 1}\right) \mathcal{L}_{\mathbf{t}}^{[1]}(\mathbf{U})\right\}\right]^{2} \\
& +\max _{1 \leq j \leq n}\left\{\left(\widehat{U}_{k 1}-U_{k 1}\right) \mathcal{L}_{\mathbf{t}}^{[11]}\left(\widetilde{\mathbf{U}}_{k}\right)+\left(\widehat{U}_{k 2}-U_{k 2}\right) \mathcal{L}_{\mathbf{t}}^{[12]}\left(\widetilde{\mathbf{U}}_{k}\right)\right\}^{2} \\
& +t_{1}^{2} \max _{1 \leq j \leq n} \mathrm{E}^{2}\left\{\mathbb{I}\left(U_{1} \geq U_{j 1}\right)-\mathbb{I}\left(U_{1} \geq \widehat{U}_{j 1}\right)\right\} .
\end{aligned}
$$

The first summand is $o_{\mathbb{P}}(1)$, uniformly in $U_{j 1}$. Because $\left|\mathcal{L}_{\mathbf{t}}^{[11]}(\mathbf{u})\right| \leq t_{1}^{2}+t_{2}^{2}$ and $\left|\mathcal{L}_{\mathbf{t}}^{[12]}(\mathbf{u})\right| \leq 2\left|t_{1} t_{2}\right|$, the second summand is $\left(t_{1}^{2}+t_{2}^{2}+2\left|t_{1} t_{2}\right|\right) O_{\mathbb{P}}(1 / n)=\left(\left|t_{1}\right|+\left|t_{2}\right|\right)^{2} O_{\mathbb{P}}(1 / n)$. Finally, the third summand is $t_{1}^{2} O_{\mathbb{P}}(1)$, and therefore $V_{n 3}^{*}(\mathbf{t})=O_{\mathbb{P}}(1 / n)\left\{\left(\left|t_{1}\right|+\left|t_{2}\right|\right)^{2}+t_{1}^{2}\right\}$. By the same arguments, one can conclude that $V_{n 4}^{\star}(\mathbf{t})=$ $O_{\mathbb{P}}(1 / n)\left\{\left(\left|t_{1}\right|+\left|t_{2}\right|\right)^{2}+t_{2}^{2}\right\}$. Hence,

$$
\begin{aligned}
V_{n}^{*}(\mathbf{t}) & =O_{\mathbb{P}}(1 / n)\left\{t_{1}^{2}+t_{2}^{2}+\left(\left|t_{1}\right|+\left|t_{2}\right|\right)^{2}+t_{1}^{2}+\left(\left|t_{1}\right|+\left|t_{2}\right|\right)^{2}+t_{2}^{2}\right\} \\
& =O_{\mathbb{P}}(1 / n)\left(t_{1}^{2}+t_{2}^{2}+\left|t_{1} t_{2}\right|\right) .
\end{aligned}
$$

Since by assumption, $\int_{\mathbb{R}^{2}}\left(t_{1}+t_{2}\right)^{4} \omega(\mathbf{t}) \mathrm{d} \mathbf{t}<\infty$,

$$
\widehat{\Delta}_{n 1, \omega}=\int_{\mathbb{R}^{2}} O_{\mathbb{P}^{*}}(1 / n)\left(t_{1}^{2}+t_{2}^{2}+\left|t_{1} t_{2}\right|\right)^{2} \omega(\mathbf{t}) \mathrm{d} \mathbf{t}+o_{\mathbb{P}^{\star}}(1)=o_{\mathbb{P}^{\star}}(1) .
$$

At this point, note that from Theorem 3.4 in [4], one deduces that as $n \rightarrow \infty$,

$$
\sup _{r \in \mathbb{R}^{+}}\left|\mathbb{P}^{\star}\left(\widetilde{S}_{n, \omega} \leq r\right)-\mathbb{P}\left(\widetilde{\mathbb{S}}_{C, \omega} \leq r\right)\right| \stackrel{\mathbb{P}}{\longrightarrow} 0,
$$

where $\widetilde{\mathbb{S}}_{C, \omega}$ has the representation given in the statement of the proposition. Since it has been shown that $\widehat{S}_{n, \omega}=\widetilde{S}_{n, \omega}+o_{\mathbb{P}^{*}}(1)$, the proof is complete.

\section{A.7 Proof of Lemma 3.2}

With $\left(I_{1}\right)_{j k}=\mathbb{I}\left(\widehat{U}_{j 1} \leq \widehat{U}_{k 1}\right)$ and $\left(I_{2}\right)_{j k}=\mathbb{I}\left(\widehat{U}_{j 2} \leq \widehat{U}_{k 2}\right)$, one can write

$$
\widehat{\Lambda}_{\mathbf{t}}\left(\widehat{\mathbf{U}}_{j}\right)=\mathcal{L}_{\mathbf{t}}\left(\widehat{\mathbf{U}}_{j}\right)+\frac{1}{n} \sum_{k=1}^{n}\left(I_{1}\right)_{j k} \mathcal{L}_{\mathbf{t}}^{[1]}\left(\widehat{\mathbf{U}}_{k}\right)+\frac{1}{n} \sum_{k=1}^{n}\left(I_{2}\right)_{j k} \mathcal{L}_{\mathbf{t}}^{[2]}\left(\widehat{\mathbf{U}}_{k}\right) .
$$


Hence,

$$
\begin{aligned}
\widehat{\Lambda}_{\mathbf{t}}\left(\widehat{\mathbf{U}}_{j}\right) \widehat{\Lambda}_{-\mathbf{t}}\left(\widehat{\mathbf{U}}_{j^{\prime}}\right)= & \mathcal{L}_{\mathbf{t}}\left(\widehat{\mathbf{U}}_{j}\right) \mathcal{L}_{-\mathbf{t}}\left(\widehat{\mathbf{U}}_{j^{\prime}}\right) \\
& +\frac{1}{n} \sum_{k=1}^{n}\left(I_{1}\right)_{j k} \mathcal{L}_{\mathbf{t}}^{[1]}\left(\widehat{\mathbf{U}}_{k}\right) \mathcal{L}_{-\mathbf{t}}\left(\widehat{\mathbf{U}}_{j^{\prime}}\right) \\
& +\frac{1}{n} \sum_{k=1}^{n}\left(I_{2}\right)_{j k} \mathcal{L}_{\mathbf{t}}^{[2]}\left(\widehat{\mathbf{U}}_{k}\right) \mathcal{L}_{-\mathbf{t}}\left(\widehat{\mathbf{U}}_{j^{\prime}}\right) \\
& +\frac{1}{n} \sum_{k=1}^{n}\left(I_{1}\right)_{j^{\prime} k} \mathcal{L}_{-\mathbf{t}}^{[1]}\left(\widehat{\mathbf{U}}_{k}\right) \mathcal{L}_{\mathbf{t}}\left(\widehat{\mathbf{U}}_{j}\right) \\
& +\frac{1}{n} \sum_{k=1}^{n}\left(I_{2}\right)_{j^{\prime} k} \mathcal{L}_{-\mathbf{t}}^{[2]}\left(\widehat{\mathbf{U}}_{k}\right) \mathcal{L}_{\mathbf{t}}\left(\widehat{\mathbf{U}}_{j}\right) \\
& +\frac{1}{n^{2}} \sum_{k, k^{\prime}=1}^{n}\left(I_{1}\right)_{j k}\left(I_{1}\right)_{j^{\prime} k^{\prime}} \mathcal{L}_{\mathbf{t}}^{[1]}\left(\widehat{\mathbf{U}}_{k}\right) \mathcal{L}_{-\mathbf{t}}^{[1]}\left(\widehat{\mathbf{U}}_{k^{\prime}}\right) \\
& +\frac{1}{n^{2}} \sum_{k, k^{\prime}=1}^{n}\left(I_{1}\right)_{j k}\left(I_{2}\right)_{j^{\prime} k^{\prime}} \mathcal{L}_{\mathbf{t}}^{[1]}\left(\widehat{\mathbf{U}}_{k}\right) \mathcal{L}_{-\mathbf{t}}^{[2]}\left(\widehat{\mathbf{U}}_{k^{\prime}}\right) \\
& +\frac{1}{n^{2}} \sum_{k, k^{\prime}=1}^{n}\left(I_{2}\right)_{j k}\left(I_{1}\right)_{j^{\prime} k^{\prime}} \mathcal{L}_{\mathbf{t}}^{[2]}\left(\widehat{\mathbf{U}}_{k}\right) \mathcal{L}_{-\mathbf{t}}^{[1]}\left(\widehat{\mathbf{U}}_{k^{\prime}}\right) \\
& +\frac{1}{n^{2}} \sum_{k, k^{\prime}=1}^{n}\left(I_{2}\right)_{j k}\left(I_{2}\right)_{j^{\prime} k^{\prime}} \mathcal{L}_{\mathbf{t}}^{[2]}\left(\widehat{\mathbf{U}}_{k}\right) \mathcal{L}_{-\mathbf{t}}^{[2]}\left(\widehat{\mathbf{U}}_{k^{\prime}}\right) .
\end{aligned}
$$

From the expression of $B_{\omega}$ in Equation (9), one has for $\ell \in\{1,2\}$ that

$$
\begin{aligned}
\int_{\mathbb{R}^{2}} \mathcal{L}_{\mathbf{t}}^{[\ell]}\left(\mathbf{u}_{1}\right) \mathcal{L}_{-\mathbf{t}}\left(\mathbf{u}_{2}\right) \omega(\mathbf{t}) \mathrm{d} \mathbf{t} & =\frac{\partial}{\partial u_{1 \ell}} \int_{\mathbb{R}^{2}} \mathcal{L}_{\mathbf{t}}\left(\mathbf{u}_{1}\right) \mathcal{L}_{-\mathbf{t}}\left(\mathbf{u}_{2}\right) \omega(\mathbf{t}) \mathrm{d} \mathbf{t} \\
& =\frac{\partial}{\partial u_{1 \ell}} B_{\omega}\left(\mathbf{u}_{1}, \mathbf{u}_{2}\right) \\
& =B_{\omega}^{[\ell]}\left(\mathbf{u}_{1}, \mathbf{u}_{2}\right),
\end{aligned}
$$

where the fact that $\mathcal{L}_{\mathbf{t}}^{[\ell]}\left(\mathbf{u}_{1}\right) \mathcal{L}_{-\mathbf{t}}\left(\mathbf{u}_{2}\right)$ is bounded, in conjunction with Lebesgue's dominated convergence theorem, have been used. Similarly, for $\ell, \ell^{\prime} \in\{1,2\}$, one obtains

$$
\int_{\mathbb{R}^{2}} \mathcal{L}_{\mathbf{t}}^{[\ell]}\left(\mathbf{u}_{1}\right) \mathcal{L}_{-\mathbf{t}}^{\left[\ell^{\prime}\right]}\left(\mathbf{u}_{2}\right) \omega(\mathbf{t}) \mathrm{d} \mathbf{t}=\frac{\partial^{2}}{\partial u_{1 \ell} \partial u_{2 \ell^{\prime}}} B_{\omega}\left(\mathbf{u}_{1}, \mathbf{u}_{2}\right)=B_{\omega}^{\left[\ell \ell^{\prime}\right]}\left(\mathbf{u}_{1}, \mathbf{u}_{2}\right) .
$$

It follows that

$$
\begin{aligned}
\left(D_{\omega}\right)_{j j^{\prime}}= & \int_{\mathbb{R}^{2}} \widehat{\Lambda}_{\mathbf{t}}\left(\widehat{\mathbf{U}}_{j}\right) \widehat{\Lambda}_{-\mathbf{t}}\left(\widehat{\mathbf{U}}_{j^{\prime}}\right) \omega(\mathbf{t}) \mathrm{d} \mathbf{t} \\
= & \left(D_{\omega, 0}\right)_{j j^{\prime}} \\
& +\frac{1}{n} \sum_{k=1}^{n}\left\{\left(I_{1}\right)_{j k}\left(D_{\omega, 1}\right)_{k j^{\prime}}+\left(I_{2}\right)_{j k}\left(D_{\omega, 2}\right)_{k j^{\prime}}+\left(I_{1}\right)_{j^{\prime} k}\left(D_{\omega, 1}\right)_{k j}+\left(I_{2}\right)_{j^{\prime} k}\left(D_{\omega, 2}\right)_{k j}\right\} \\
& +\frac{1}{n^{2}} \sum_{k, k^{\prime}=1}^{n}\left\{\left(I_{1}\right)_{j k}\left(D_{\omega, 11}\right)_{k k^{\prime}}\left(I_{1}\right)_{k^{\prime} j^{\prime}}+\left(I_{1}\right)_{j k}\left(D_{\omega, 12}\right)_{k k^{\prime}}\left(I_{2}\right)_{k^{\prime} j^{\prime}}\right\} \\
& +\frac{1}{n^{2}} \sum_{k, k^{\prime}=1}^{n}\left\{\left(I_{2}\right)_{j k}\left(D_{\omega, 21}\right)_{k k^{\prime}}\left(I_{1}\right)_{k^{\prime} j^{\prime}}+\left(I_{2}\right)_{j k}\left(D_{\omega, 22}\right)_{k k^{\prime}}\left(I_{2}\right)_{k^{\prime} j^{\prime}}\right\} \\
= & \left(D_{\omega, 0}\right)_{j j^{\prime}}+\frac{1}{n}\left\{\left(I_{1} D_{\omega, 1}\right)_{j j^{\prime}}+\left(I_{2} D_{\omega, 2}\right)_{j j^{\prime}}+\left(I_{1} D_{\omega, 1}\right)_{j^{\prime} j}+\left(I_{2} D_{\omega, 2}\right)_{j^{\prime} j}\right\} \\
& +\frac{1}{n^{2}}\left\{\left(I_{1} D_{\omega, 11} I_{1}^{\top}\right)_{j j^{\prime}}+\left(I_{1} D_{\omega, 12} I_{2}^{\top}\right)_{j j^{\prime}}+\left(I_{2} D_{\omega, 21} I_{1}^{\top}\right)_{j j^{\prime}}+\left(I_{2} D_{\omega, 22} I_{2}^{\top}\right)_{j j^{\prime}}\right\} .
\end{aligned}
$$


Finally,

$$
\begin{aligned}
D_{\omega}= & D_{\omega, 0}+\frac{1}{n}\left(I_{1} D_{\omega, 1}+I_{2} D_{\omega, 2}+\left(I_{1} D_{\omega, 1}\right)^{\top}+\left(I_{2} D_{\omega, 2}\right)^{\top}\right) \\
& +\frac{1}{n^{2}}\left(I_{1} D_{\omega, 11} I_{1}^{\top}+I_{1} D_{\omega, 12} I_{2}^{\top}+I_{2} D_{\omega, 21} I_{1}^{\top}+I_{2} D_{\omega, 22} I_{2}^{\top}\right) .
\end{aligned}
$$

\section{B Details on a test by [5]}

Let $B \in \mathbb{R}^{n \times n}$ such that for each $j, k \in\{1, \ldots, n\}$,

$$
B_{j k}=\mathbb{I}\left(\widehat{U}_{j 1} \leq \widehat{U}_{k 1}, \widehat{U}_{j 2} \leq \widehat{U}_{k 2}\right)-\mathbb{I}\left(\widehat{U}_{j 1} \leq \widehat{U}_{k 2}, \widehat{U}_{j 2} \leq \widehat{U}_{k 1}\right) .
$$

One can then write for $\mathbf{1}=(1, \ldots, 1) \in \mathbb{R}^{n}$ that

$$
\begin{aligned}
S_{n}^{\mathrm{CvM}}=\sum_{k=1}^{n}\left(\frac{1}{n} \sum_{j=1}^{n} B_{j k}\right)^{2} & =\frac{1}{n^{2}} \sum_{k=1}^{n} \sum_{j, j^{\prime}=1}^{n} B_{j k} B_{k j^{\prime}}^{\top} \\
& =\frac{1}{n^{2}} \sum_{j, j^{\prime}=1}^{n}\left(B B^{\top}\right)_{j j^{\prime}}=\frac{1}{n^{2}} \mathbf{1}\left(B B^{\top}\right) \mathbf{1}^{\top} .
\end{aligned}
$$

It was shown by [5] that $S_{n}^{\mathrm{CvM}}$ converges in distribution under $\mathbb{H}_{0}$ to a random variable having representation

$$
\mathbb{S}^{\mathrm{CvM}}=\int_{[0,1]^{2}}\left\{\mathbb{E}\left(u_{1}, u_{2}\right)\right\}^{2} \mathrm{~d} C\left(u_{1}, u_{2}\right),
$$

where in terms of a $C$-Brownian sheet $\mathbb{B}_{C}$ on $[0,1]^{2}$ and for $\dot{C}_{1}\left(u_{1}, u_{2}\right)=\partial C\left(u_{1}, u_{2}\right) / \partial u_{1}$ and $\dot{C}_{2}\left(u_{1}, u_{2}\right)=$ $\partial C\left(u_{1}, u_{2}\right) / \partial u_{2}$,

$$
\mathbb{E}\left(u_{1}, u_{2}\right)=\widetilde{\mathbb{B}}_{C}\left(u_{1}, u_{2}\right)-\dot{C}_{1}\left(u_{1}, u_{2}\right) \widetilde{\mathbb{B}}_{C}\left(u_{1}, 1\right)-\dot{C}_{2}\left(u_{1}, u_{2}\right) \widetilde{\mathbb{B}}_{C}\left(1, u_{2}\right),
$$

where $\widetilde{\mathbb{B}}_{C}\left(u_{1}, u_{2}\right)=\mathbb{B}_{C}\left(u_{1}, u_{2}\right)-\mathbb{B}_{C}\left(u_{2}, u_{1}\right)$. Letting

$$
\widehat{\mathbb{B}}_{C}\left(u_{1}, u_{2}\right)=\frac{1}{\sqrt{n}} \sum_{j=1}^{n} \Delta_{j}\left\{\mathbb{I}\left(\widehat{U}_{j 1} \leq u_{1}, \widehat{U}_{j 2} \leq u_{2}\right)-\mathbb{I}\left(\widehat{U}_{j 1}<u_{2}, \widehat{U}_{j 2}<u_{1}\right)\right\},
$$

the multiplier version of $\mathbb{E}$ is given by

$$
\widehat{\mathbb{E}}\left(u_{1}, u_{2}\right)=\widehat{\mathbb{B}}_{C}\left(u_{1}, u_{2}\right)-\dot{C}_{1 n}\left(u_{1}, u_{2}\right) \widehat{\mathbb{B}}_{C}\left(u_{1}, 1\right)-\dot{C}_{2 n}\left(u_{1}, u_{2}\right) \widehat{\mathbb{B}}_{C}\left(1, u_{2}\right) .
$$

In the last expression,

$$
\dot{C}_{1 n}\left(u_{1}, u_{2}\right)= \begin{cases}\frac{C_{n}\left(2 \ell_{n}, u_{2}\right)}{2 \ell_{n}}, & \text { if } u_{1} \in\left[0, \ell_{n}\right), \\ \frac{C_{n}\left(u_{1}+\ell_{n}, u_{2}\right)-C_{n}\left(u_{1}-\ell_{n}, u_{2}\right)}{2 \ell_{n}}, & \text { if } u_{1} \in\left[\ell_{n}, 1-\ell_{n}\right], \\ \frac{C_{n}\left(1, u_{2}\right)-C_{n}\left(1-2 \ell_{n}, u_{2}\right)}{2 \ell_{n}}, & \text { if } u_{1} \in\left(1-\ell_{n}, 1\right] .\end{cases}
$$

is a finite-difference estimator of the partial derivative $\dot{C}_{1}$ in term of $\ell_{n} \in(0,1 / 2) ; \dot{C}_{2}$ is estimated similarly. Finally, the multiplier version of $S_{n}^{\mathrm{CvM}}$ is given by

$$
\widehat{S}_{n}^{\mathrm{CvM}}=\int_{[0,1]^{2}}\left\{\widehat{\mathbb{E}}\left(u_{1}, u_{2}\right)\right\}^{2} \mathrm{~d} C_{n}\left(u_{1}, u_{2}\right)=\frac{1}{n} \sum_{k=1}^{n}\left\{\widehat{\mathbb{E}}\left(\widehat{U}_{k 1}, \widehat{U}_{k 2}\right)\right\}^{2} .
$$


Now define $\widetilde{B} \in \mathbb{R}^{n \times n}$ such that for each $j, k \in\{1, \ldots, n\}$,

$$
\begin{aligned}
\widetilde{B}_{j k}= & \mathbb{I}\left(\widehat{U}_{j 1} \leq \widehat{U}_{k 1}, \widehat{U}_{j 2} \leq \widehat{U}_{k 2}\right)-\mathbb{I}\left(\widehat{U}_{j 1} \leq \widehat{U}_{k 2}, \widehat{U}_{j 2} \leq \widehat{U}_{k 1}\right) \\
& -\dot{C}_{1 n}\left(\widehat{U}_{k 1}, \widehat{U}_{k 2}\right)\left\{\mathbb{I}\left(\widehat{U}_{j 1} \leq \widehat{U}_{k 1}\right)-\mathbb{I}\left(\widehat{U}_{j 2} \leq \widehat{U}_{k 1}\right)\right\} \\
& -\dot{C}_{2 n}\left(\widehat{U}_{k 1}, \widehat{U}_{k 2}\right)\left\{\mathbb{I}\left(\widehat{U}_{j 2} \leq \widehat{U}_{k 2}\right)-\mathbb{I}\left(\widehat{U}_{j 1} \leq \widehat{U}_{k 2}\right)\right\}
\end{aligned}
$$

With this notation, one can show that

$$
\widehat{S}_{n}^{\mathrm{CvM}}=\frac{1}{n} \sum_{k=1}^{n}\left(\frac{1}{\sqrt{n}} \sum_{j=1}^{n} \Delta_{j} \widetilde{B}_{j k}\right)^{2}=\frac{1}{n^{2}} \boldsymbol{\Delta}\left(\widetilde{B} \widetilde{B}^{\top}\right) \boldsymbol{\Delta}^{\top} .
$$

\section{References}

[1] Bahraoui, T., T. Bouezmarni, and J.-F. Quessy (2018). A family of goodness-of-fit tests for copulas based on characteristic functions. Scand. J. Stat. 45(2), 301-323.

[2] Bahraoui, T. and J.-F. Quessy (2017). Tests of radial symmetry for multivariate copulas based on the copula characteristic function. Electron. J. Stat. 11(1), 2066-2096.

[3] Bücher, A. and H. Dette (2010). A note on bootstrap approximations for the empirical copula process. Statist. Probab. Lett. 80(23-24), 1925-1932.

[4] Dehling, H. and T. Mikosch (1994). Random quadratic forms and the bootstrap for U-statistics. J. Multivariate Anal. 51(2), 392-413.

[5] Genest, C., J. Nešlehová, and J.-F. Quessy (2012). Tests of symmetry for bivariate copulas. Ann. Inst. Statist. Math. 64(4), 811-834.

[6] Genest, C. and J. G. Nešlehová (2014). On tests of radial symmetry for bivariate copulas. Statist. Papers 55(4), $1107-1119$.

[7] Harder, M. and U. Stadtmüller (2017). Testing exchangeability of copulas in arbitrary dimension. J. Nonparametr. Stat. 29(1), 40-60.

[8] Jiménez-Gamero, M., V. Alba-Fernández, J. Muñoz-García, and Y. Chalco-Cano (2009). Goodness-of-fit tests based on empirical characteristic functions. Comput. Statist. Data Anal. 53(12), 3957-3971.

[9] Khoudraji, A. (1995). Contributions à l'étude des Copules et à la Modélisation de Valeurs Extrêmes Bivariées. PhD thesis, Université Laval, Canada.

[10] Kosorok, M. (2008). Introduction to Empirical Processes and Semiparametric Inference. Springer, New York.

[11] Lee, A. J. (1990). U-Statistics: Theory and Practice. Marcel Dekker, New York.

[12] Leucht, A. and M. H. Neumann (2013). Dependent wild bootstrap for degenerate $U$ - and $V$-statistics. J. Multivariate Anal. 117 , 257-280.

[13] Mai, J.-F. and M. Scherer (2012). Simulating Copulas: Stochastic Models, Sampling Algorithms, and Applications. Imperial College Press, London.

[14] Nelsen, R. B. (2006). An Introduction to Copulas. Second edition. Springer, New York.

[15] Nelsen, R. B. (2007). Extremes of nonexchangeability. Statist. Papers 48(2), 329-336.

[16] Quessy, J.-F. (2016). A general framework for testing homogeneity hypotheses about copulas. Electron. J. Stat. 10(1), 10641097.

[17] Quessy, J.-F. and O. Kortbi (2016). Minimum-distance statistics for the selection of an asymmetric copula in Khoudraji's class of models. Statist. Sinica 26(1), 177-204.

[18] Quessy, J.-F., L.-P. Rivest, and M.-H. Toupin (2016). On the family of multivariate chi-square copulas. J. Multivariate Anal. 152, 40-60.

[19] Shorack, G. R. and J. A. Wellner (1986). Empirical Processes with Applications to Statistics. John Wiley \& Sons, New York. 